\title{
Reduced thyroxine production in young household contacts of tuberculosis patients increases active tuberculosis disease risk
}

\author{
Kamakshi Prudhula Devalraju, ${ }^{1}$ Deepak Tripathi, ${ }^{2}$ Venkata Sanjeev Kumar Neela, ${ }^{1}$ \\ Padmaja Paidipally, ${ }^{2}$ Rajesh Kumar Radhakrishnan, ${ }^{2}$ Karan P. Singh, ${ }^{3}$ Mohammad Soheb Ansari, ${ }^{1}$ \\ Martin Jaeger, ${ }^{4,5}$ Romana T. Netea-Maier, ${ }^{4}$ Mihai G. Netea, ${ }^{5}$ Sunmi Park, ${ }^{6}$ Sheue-yann Cheng, ${ }^{6}$ \\ Vijaya Lakshmi Valluri,' and Ramakrishna Vankayalapati \\ 1'Immunology and Molecular Biology Department, Bhagwan Mahavir Medical Research Centre, Hyderabad, Telangana, \\ India. ${ }^{2}$ Department of Pulmonary Immunology, Center for Biomedical Research, University of Texas Health Science Center, \\ Tyler, Texas, USA. ${ }^{3}$ Department of Epidemiology and Biostatistics, School of Community and Rural Health, University \\ of Texas Health Science Center, Tyler, Texas, USA. ${ }^{4}$ Department of Internal Medicine, Division of Endocrinology, and \\ ${ }^{5}$ Department of Internal Medicine and Radboud Center for Infectious Diseases, Radboud University Medical Center, \\ Nijmegen, Netherlands. ${ }^{\circ}$ Laboratory of Molecular Biology, National Cancer Institute, NIH, Bethesda, Maryland, USA.
}

In the current study, we followed 839 household contacts (HHCs) of tuberculosis (TB) patients for 2 years and identified the factors that enhanced the development of TB. Fourteen of the $17 \mathrm{HHCs}$ who progressed to TB were in the 15- to 30 -year-old age group. At baseline (the " 0 " time point, when all the individuals were healthy), the concentration of the thyroid hormone thyroxine (T4) was lower, and there were increased numbers of Tregs in PBMCs of TB progressors. At baseline, PBMCs from TB progressors stimulated with early secretory antigenic target 6 (ESAT-6) and 10 kDa culture filtrate antigen (CFP-10) produced less IL-1 $\alpha$. Thyroid hormones inhibited Mycobacterium tuberculosis (Mtb) growth in macrophages in an IL-1 $\alpha$-dependent manner. Mtb-infected Thra1 ${ }^{\mathrm{PV} /+}$ (mutant thyroid hormone receptor) mice had increased mortality and reduced IL-1 $\alpha$ production. Our findings suggest that young HHCs who exhibit decreased production of thyroid hormones are at high risk of developing active TB disease.

Authorship note: KPD, DT, and VSKN contributed equally to this work. VLV and RV are co-senior authors.

Conflict of interest: The authors have declared that no conflict of interest exists.

Copyright: () 2021, Devalraju et al. This is an open access article published under the terms of the Creative Commons Attribution 4.0 International License.

Submitted: February 2, 2021

Accepted: May 26, 2021

Published: July 8, 2021

Reference information: /CI Insight. 2021;6(13):e148271.

https://doi.org/10.1172/jci. insight.148271.

\section{Introduction}

Mycobacterium tuberculosis (Mtb) infects one-third of humans and causes almost 1.3 million deaths per year (1). Approximately $90 \%$ of infected persons have latent tuberculosis infection (LTBI) and remain well, but $10 \%$ develop primary tuberculosis (TB) soon after infection or reactivation of TB many years later $(2,3)$. Nevertheless, only a small percentage of persons with LTBI develop TB and therefore are required to complete treatment regimens; approximately $13 \%-42 \%$ of individuals prescribed treatment, fail to complete therapy $(4,5)$. The identification of persons with LTBI who are at greatly increased risk of development of TB would be a significant breakthrough that would allow public health resources to be focused on highrisk individuals, facilitate the completion of therapy for LTBI, and prevent the future development of TB. Severe immunosuppression due to HIV infection and treatment with corticosteroids and anti-TNF blockers markedly increase the risk of the progression of LTBI to active TB (6-9). Among persons who are not clinically immunocompromised, limited information is available about other immune mechanisms that favor the progression of LTBI to TB (10-21).

The household contacts (HHCs) of TB patients are at high risk of developing latent Mtb infection or active TB disease $(22,23)$. Identifying the HHCs that are likely to develop active TB is important to control TB (24-26). Examination of HHCs is a natural method to study active TB disease development in a realtime manner and to explore the early immune components involved in protection against TB. However, a detailed follow-up study and mechanistic understanding are important to identify the healthy HHCs of TB patients who are at high risk. 
Immune responses mediated by antigen-presenting cells, NK cells, and T cells play an important role in the control of Mtb infection (27). This is partly achieved through the production of cytokines such as IL-1 $\alpha$, TNF- $\alpha$, IFN- $\gamma$, and IL-1 $\beta$ in the lungs of Mtb-infected mice and independently required for host resistance (28). TNF- $\alpha$-mediated signaling is critical for reactive nitrogen production in macrophages and protection against Mtb (29). IFN- $\gamma$ induces macrophage activation and controls growth of intracellular pathogens.

Previous studies have demonstrated that monocytes, NK cells, innate lymphoid cells, and Tregs have the potential to contribute to innate and adaptive immune responses during Mtb infection (30-33). Endocrine abnormalities such as adrenal insufficiency, diabetes mellitus, and calcium-vitamin D abnormalities are common in TB patients, suggesting that the endocrine system also plays an important role in the control of Mtb infection (34-36). Hormones can affect immune cell function and induce the production of inflammatory cytokines. In a rabbit Mtb infection model, it was shown that defective thyroid hormone production enhances susceptibility to Mtb infection (37). However, it is not known whether defective hormone production leads to the development of active TB disease in HHCs of TB patients.

In the current study, we evaluated the phenotype and function of various immune cells and other factors, such as hormones, in a large group of household contacts of TB patients (a total of 839 individuals). We repeated these evaluations at 24 months while monitoring the subjects for the development of active TB. By following a large cohort of HHCs of TB patients, we determined whether the development of TB is preceded by specific changes in the cellular markers of immune cells and other factors. We also confirmed our findings using a mouse model of Mtb infection.

\section{Results}

Demographic characteristics of the cohort. Among the 839 individuals enrolled, we had $83.5 \%$ retention, and 138 individuals were lost to follow-up. The median number of HHCs per index case was 3. Fifty-nine percent of the HHCs were LTBI-positive at baseline, and $2.53 \%$ of the HHCs progressed to active TB disease during follow-up. Among the $839 \mathrm{HHCs}, 465$ (56\%) were 15-30 years old, 228 (27\%) were 31-44 years old, and 146 (17\%) were 45-73 years old (Table 1). Demographic and clinical characteristics of the study participants are shown in Table 1 . The summaries of screening, enrollment, and loss to follow-up are shown in Supplemental Tables 1 and 2; supplemental material available online with this article; https:// doi.org/10.1172/jci.insight.148271DS1.

The prevalence of TB was high in young HHCs. During the 2-year follow-up period, 688 HHCs successfully completed the study. Seventeen (2.53\%) out of 688 HHCs developed active TB (progressors), and 671 of the HHCs remained healthy (nonprogressors) (Figure 1 and Table 1). Among the 17 progressors, $14(82.35 \%)$ were in the 15-30-year-old age group, $2(11.8 \%)$ were in the $31-44$-year-old age group, and 1 (5.9\%) was in the 45-73-year-old age group (Supplemental Table 3). In summary, young HHCs of TB index cases were at increased risk of developing active TB (relative risk, 3.75; 95\% CI, 1.086 to $12.96 ; P$ $=0.036$ ). The demographic and clinical characteristics of the HHCs that developed active TB during the 2-year study are shown in Supplemental Table 3. The complete blood cell counts of the progressors and nonprogressors are shown in Supplemental Figure 1.

High $C D 16^{+} C D 56^{+}$and Treg cell frequencies in progressors at baseline. We determined the percentages of various immune cell populations in freshly isolated PBMCs of subsets of nonprogressors $(n=87)$ and progressors $(n=12)$ at baseline and during the follow-up visits. The frequencies of $\operatorname{CD}_{14}^{+}(P=\mathrm{NS}$, Figure 2A and Supplemental Figure 2$)$ and $\mathrm{CD} 14^{+} \mathrm{CD} 16^{+}$cells $(P=\mathrm{NS}$, Figure $2 \mathrm{~B}$ and Supplemental Figure 2$)$ were similar at baseline in the progressors compared with the nonprogressors. The percentages of $\mathrm{CD} 16^{+} \mathrm{CD} 56^{+}$cells were higher at baseline in the progressors than in the nonprogressors $(P=0.035$; Figure $2 \mathrm{C}$ and Supplemental Figure 2). Interestingly, there was a 2.5 -fold decrease in the percentage of $\mathrm{CD} 16^{+} \mathrm{CD} 56^{+}$cells $(P=0.0006$; Figure $2 \mathrm{C}$ ) in the progressors at follow-up (blood was obtained when they developed active TB) compared with the baseline values. The percentages of $\mathrm{CD} 4{ }^{+} \mathrm{CD} 25^{+} \mathrm{FoxP} 3^{+}$cells (Tregs) were significantly higher in the progressors at baseline $(P=0.048)$ and follow-up $(P=0.047$; Figure $2 \mathrm{H}$ and Supplemental Figure 2$)$ than in nonprogressors. No significant differences were observed in percentages of $\mathrm{NK}$ cells and $\mathrm{T}$ cells in progressors compared to nonprogressors (Figure 2, D-G).

Reduced IL-1 $\alpha$ production by PBMCs in progressors at baseline. We determined cytokine and chemokine production by freshly isolated PBMCs from progressors $(n=12)$ and nonprogressors $(n=12)$ cultured in the presence and absence of the Mtb antigens early secretory antigenic target 6 (ESAT-6) and culture filtrate antigen (CFP-10), as mentioned in the Methods. Among 34 cytokines and chemokines tested, as shown in 
Table 1. Demographic characteristics of the cohort.

\begin{tabular}{lcc}
\hline Characteristics & & $N(\%)$ \\
Cohort size & & 839 \\
Median age (years) & $15-30$ years & $28(15-73)$ \\
HHCs enrolled in different age group & $31-44$ years & $465(56 \%)$ \\
& $45-73$ years & $228(27 \%)$ \\
Sex & Male & $146(17 \%)$ \\
Smokers & Female & $49.4 \%$ \\
Alcoholism & & $50.6 \%$ \\
Screening history & Chest x-rays obtained & $73(8.61 \%)$ \\
\hline Outcome group & HIV test obtained & $111(13.2 \%)$ \\
BCC vaccination (percentage) & Completed study & 15 \\
Prevalence of latent TB infection in the cohort & Lost to follow-up & 739 \\
Active TB converters & & 138 \\
Median/mean size of the household & & $479(69 \%)-$ vaccination \\
HIV prevalence in cohort (percentage) & & $495(59 \%)$ \\
Other comorbidities & & $17(2.53 \%)$ \\
Minimum duration of stay with the index & & 3 \\
patient (months) & & $N=1(0.12 \%)$ \\
\hline
\end{tabular}

Figure 3 and Supplemental Figure 3, at baseline, the production of IFN- $\gamma$, IL-13, IL-10, and IL-18 was higher in the culture supernatants of the progressors than the nonprogressors $(P<0.05$, Figure 3$)$. In contrast, IL- $1 \alpha$ $(P=0.047$; Figure 3$)$ levels were significantly lower in the culture supernatants of the progressors compared with the supernatants of the nonprogressors. At follow-up, IFN- $\gamma(P=0.09)$, IL-17, IL-22, IL-23, and IL-1 $\alpha$ $(P<0.05)$ levels were lower in the progressors than in nonprogressors (Figure 3 and Supplemental Figure 3$)$.

Lower hormone levels in the progressors at baseline. The findings in Supplemental Table 3 indicated that 14 out of the 17 HHCs who developed active TB disease were in the 15- to 30-year-old age group. Given that hormones play an important role in growth, metabolism, and immunity (38), we determined the levels of various serum hormones in the progressors and nonprogressors (both in the same age group) at baseline (Figure 4 and Supplemental Figure 4). Serum dehydroepiandrosterone (DHEA), cortisol, and thyroid stimulating hormone (TSH) levels were similar in progressors and nonprogressors at baseline (Supplemental Figure 4). As shown in Figure 4B, at baseline, the level of thyroxine (T4) hormone $(P=0.0002)$ was significantly lower in the serum of the progressors compared with the nonprogressors (relative risk, 7.3; 95\% CI, 3.09 to $17.35 ; P=0.0001)$. There was a further decrease in the T4 levels during TB activation $(P=0.003)$ compared with baseline. After standard antitubercular treatment (ATT), serum triiodothyronine (T3) and T4 significantly increased in progressors (Figure 4, A and B). However, 3 years after ATT, the level of T4 hormones significantly decreased in treated progressors (Figure 4B). The diagnostic accuracies of various hormones were evaluated by receiver operating characteristic (ROC) curve analysis to distinguish progressors and nonprogressors at baseline. ROC curve analysis revealed that among all the measured hormones, baseline T4 (AUC > 0.86) and DHEA levels (AUC > 0.78) could distinguish between nonprogressor and progressor HHCs, with a high AUC value (Figure 4C and Supplemental Figure 5, A-C).

Monocytes/monocyte-derived macrophages upregulate thyroid hormone receptors in response to $\gamma$-Mtb stimulation. We determined the expression of thyroid (TR1 $\alpha$ and TR1 $\beta$ ) and steroid hormone receptors (glucocorticoid receptor [GR1]) by various immune cell populations within freshly isolated PBMCs before and after stimulation with $\gamma$-irradiated $\mathrm{Mtb}(\gamma$-Mtb). As shown in Figure 5, A and B and Supplemental Figure 8, TR1 $\alpha$, TR1 $\beta$, and glucocorticoid receptor expression were significantly higher in monocytes than in NK, T, NKT, and B cells from fresh PBMCs. $\gamma$-Mtb significantly enhanced TR1 $\alpha$, TR1 $\beta$, and glucocorticoid receptor expression by macrophages compared with NK cells, B cells, and T cells (Figure 5, A and B, and Supplemental Figures 6-8). In $\gamma$-Mtb-stimulated PBMCs, we also found higher expression of TR1 $\alpha$ on NK and T cells compared with unstimulated PBMCs (Figure 5A). Further, Mtb H37Rv infection enhanced the 


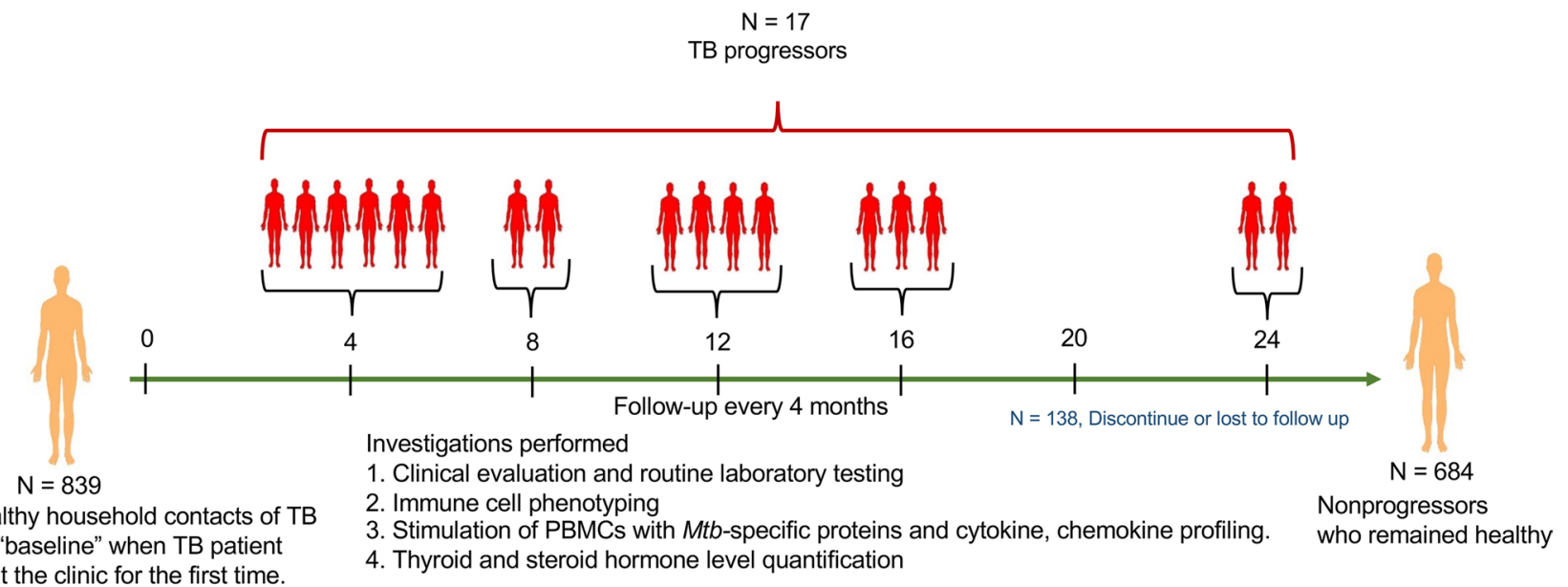
patients at "baseline" when TB patient was seen at the clinic for the first time.

4. Thyroid and steroid hormone level quantification

Figure 1. Study design and outcomes for the household contacts in the TB patient cohort study. Schematic representation of the experimental design.

expression of thyroid hormone receptor TR $\alpha 1 / \beta 1$ complex on human monocytes/monocyte-derived macrophages (MDMs) $(P<0.0001$, Figure 6, B and C). Deiodinase (DIO) type 2 (D2) converts prohormone T4 into the active hormone $\mathrm{T} 3$ in macrophages and plays a crucial role in cytokine production and phagocytosis $(39,40)$. Control and Mtb H37Rv-infected human MDMs expressed DIO 1, 2, and 3, as determined by Western immunoblotting (Supplemental Figure 9).

$T 3$ and T4 hormones restrict Mtb growth in human MDMs. We determined the effects of thyroid and steroid hormones on intracellular mycobacterial growth in MDMs. Freshly prepared MDMs from 7 healthy individuals aged 18-30 years were infected with Mtb H37Rv at an MOI of 2.5 and cultured in the presence or absence of various concentrations of the hormones T3, T4, cortisol, and DHEA. After 120 hours, $4.6 \times 10^{5} \pm$ $0.54 \times 10^{5} \mathrm{CFUs}$ per well of untreated MDMs were present. Addition of T3 $(15 \mathrm{nmol} / \mathrm{L})$ and T4 $(150 \mathrm{nmo}-$ $1 / \mathrm{L}$ ) hormones significantly reduced the number of CFUs in the MDMs (from $4.6 \times 10^{5} \pm 0.54 \times 10^{5}$ to 6.7 $\times 10^{4} \pm 0.21 \times 10^{4}, P<0.0001$, for T3 and $1.7 \times 10^{5} \pm 0.76 \times 10^{5}, P=0.0006$, for T4) (Figure 5C). However, supplementation with the cortisol and DHEA hormones had no effect on Mtb growth in MDMs (Figure 5C). The viability of the hormone-treated and untreated MDMs was similar (Supplemental Figure 10). Our results demonstrated that thyroid hormones inhibited Mtb growth in MDMs more efficiently than steroid hormones.

Hormones enhance IL-1 $\alpha$ production by Mtb-infected MDMs. To determine the mechanism(s) by which T3 and T4 hormones inhibit Mtb growth in human MDMs, we measured various cytokine and chemokine levels in the culture supernatants of Mtb-infected MDMs by multiplex ELISA. Freshly prepared MDMs from 5 healthy individuals from the 18- to 30-year-old age group were infected with Mtb $\mathrm{H} 37 \mathrm{Rv}$ at an MOI of 2.5 and cultured in the presence or absence of T3 $(15 \mathrm{nmol} / \mathrm{L})$ and T4 $(150 \mathrm{nmol} / \mathrm{L})$. Addition of T3 and T4 hormones to Mtb-infected MDMs enhanced the production of IL-1 $\alpha(P=0.0008$ in the presence or absence of T3; $P=0.048$ in the presence or absence of T4) (Figure 6A and Supplemental Figure 11). We also confirmed the above findings with confocal microscopy, and the H37Rv-infected human MDMs produced more IL- $1 \alpha$ in the presence of the T4 hormone (Figure 6C).

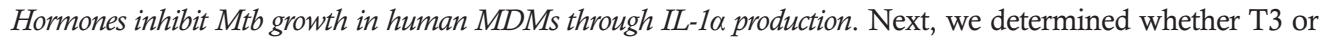
T4 hormone-mediated IL-1 $\alpha$ production restricts Mtb growth in human MDMs. Freshly prepared MDMs from 6 healthy individuals aged $18-30$ years were infected with Mtb H37Rv and cultured in the presence of T4 hormone. Some of the T4 hormone-treated and untreated wells were treated with anti-IL-1 $\alpha$ antibody. As shown in Figure 6D, after 120 hours, $4.1 \times 10^{5} \pm 1.07 \times 10^{5} \mathrm{CFUs}$ per well of untreated MDMs were present. Addition of T4 hormone reduced the number of CFUs to $1.2 \times 10^{5} \pm 0.20 \times 10^{5}(P<0.0001$; Figure $6 \mathrm{D})$. The addition of the anti-IL-1 $\alpha$ antibody enhanced Mtb growth in T4 hormone-treated wells $\left(1.2 \times 10^{5} \pm 1.07 \times 10^{5}\right.$ to 2.5 $\times 10^{5} \pm 0.51 \times 10^{5} \mathrm{CFU}, P=0.03$; Figure 6D). The treatment of Mtb-infected MDMs with recombinant IL-1 $\alpha$ also restricted Mtb growth (from $3.8 \times 10^{5} \pm 0.51 \times 10^{5}$ to $1.5 \times 10^{5} \pm 0.72 \times 10^{5} \mathrm{CFUs}, P=0.004$; Figure $6 \mathrm{E}$ ).

Thra $1^{P V /+}$ mice are susceptible to Mtb infection. To determine the role of thyroid hormone signaling in Mtb infection, we infected $\mathrm{WT}$ and Thra $1^{\mathrm{PV} /+}$ mice expressing a dominant-negative thyroid receptor $\alpha 1$ mutant (TRa1PV) with Mtb H37Rv as described in the Methods (Supplemental Figure 12 and Figure 7A) (41). 
A

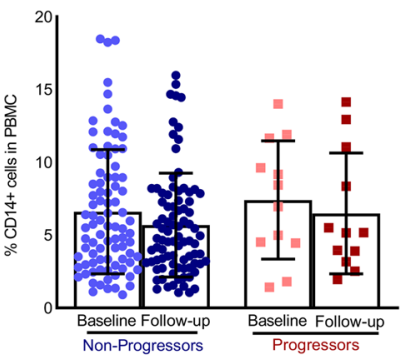

E CCR7+ Memory like NK cells

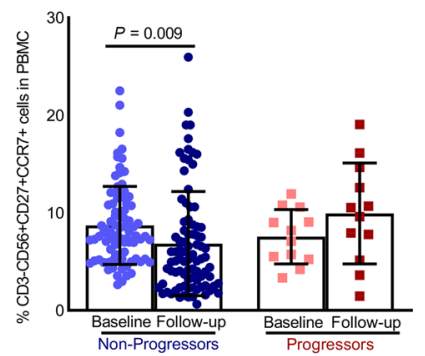

B

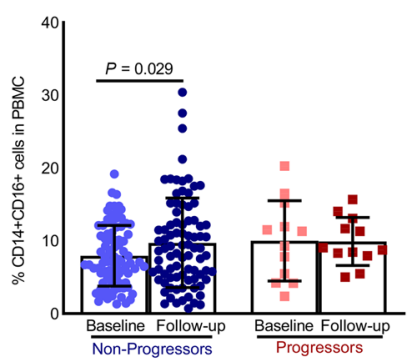

$\mathbf{F}$

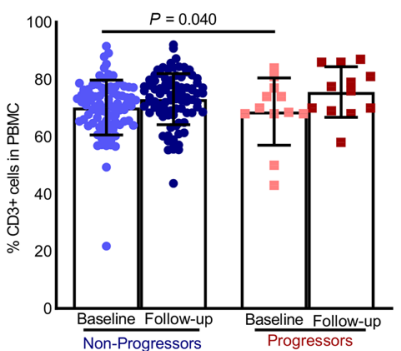

C $\mathrm{CD} 16+$ expressing $\mathrm{CD} 56+$ cells

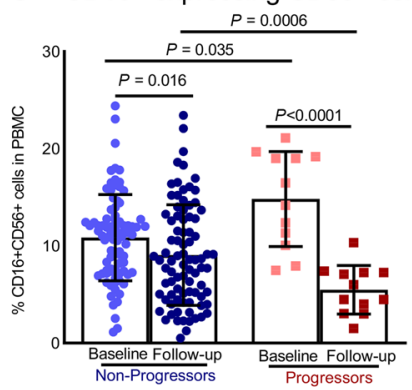

CD4+ T cells

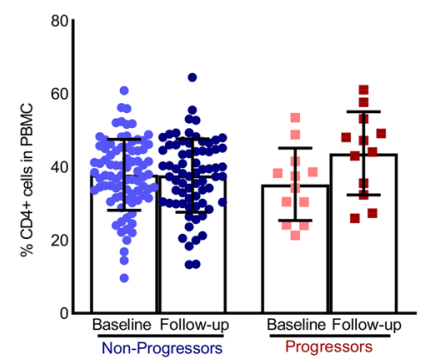

D

NK cells

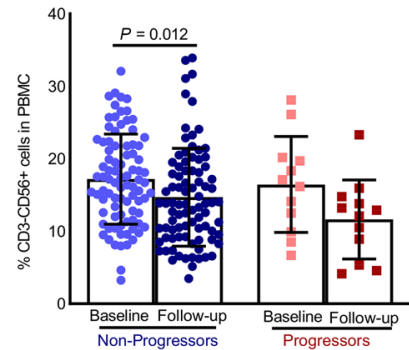

H Tregs

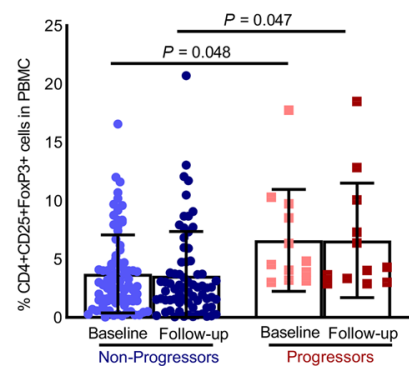

Figure 2. Immune cell distribution in the PBMCs of household contacts of TB patients. PBMCs were isolated from age-matched non-TB progressors $(n=$ 87) and TB progressors $(n=12)$ at baseline and follow-up (when the progressors were registered as having active TB), and the percentages of (A) CD14 ${ }^{+}$, (B)

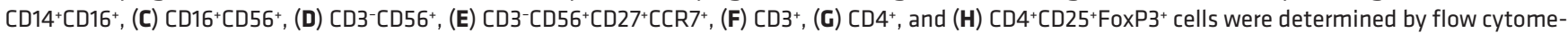
try. The data from 87 nonprogressors and 12 progressors are shown. All the age-matched, non-TB progressors were healthy, nonsmoking, and nonalcoholic and were without any immunosuppressive conditions at baseline or follow-up. Data from smoking and alcoholic progressors were not included. $P$ values were determined using 1-way ANOVA with Tukey's multiple-comparison test. The mean \pm SEM and $P$ values are shown.

$\mathrm{Thra}^{\mathrm{PV} /+}$ mice were dwarfs, and by 6 weeks of age, the mean body weight of Thra $1^{\mathrm{PV} /+}$ mice was $12.4 \pm$ $2.9 \mathrm{~g}$, which was approximately 40\% less than that of their WT littermates (Supplemental Figure 13) (41). Mtb infection significantly reduced the weight of Thra $1^{\mathrm{PV} /+}$ mice compared with that of uninfected WT or Thra $1^{\mathrm{PV} /+}$ mice (Supplemental Figure 13). As shown in Figure 7B, by 65 days after infection, 44.5\% of the Mtb-infected Thra $1^{\mathrm{PV} /+}$ mice had died, whereas only $25 \%$ of the uninfected Thra $1^{\mathrm{PV}+}$ mice had died. In contrast, all WT-infected or uninfected mice survived (Figure 7B). One month after Mtb infection, there was a significant increase in the number of CFUs of bacteria in the lungs of Thra1 ${ }^{\mathrm{PV}+}$ mice compared with WT mice infected with $\mathrm{Mtb}\left(1.8 \times 10^{6} \pm 0.22 \times 10^{6}\right.$ vs. $0.45 \times 10^{6} \pm 0.047 \times 10^{6} \mathrm{CFUs}, P=0.0004$, Figure $\left.7 \mathrm{C}\right)$.

Mtb-infected Thra ${ }^{P V /+}$ mice have defective immune responses. We asked whether lack of thyroid hormone signaling had any effect on immune responses during Mtb infection. WT and Thra $1^{\mathrm{PV} /+}$ mice were infected with $\mathrm{Mtb}$, and after 1 month the levels of various cytokines and chemokines were measured in lung homogenates by multiplex (34-plex) ELISA. As shown in Figure 7D and Supplemental Figure 14, levels of inflammatory cytokines IFN- $\gamma$ and IL- $1 \alpha$ and chemokines IP-10, MIP-1 $\alpha$, MCP-3, and MIP-1 $\beta$ were significantly lower in whole lung homogenates of Mtb-infected Thra $1^{\mathrm{PV} /+}$ mice than in homogenates of Mtb-infected WT mice (Figure 7D). We also examined various leukocyte populations by flow cytometry. The absolute numbers of total $\mathrm{CD} 45^{+}$and $\mathrm{CD} 45^{+} \mathrm{F} 4 / 80^{+}$cells were significantly lower, whereas the numbers of Tregs $\left(\mathrm{CD} 4{ }^{+} \mathrm{CD} 25^{+} \mathrm{FoxP} 3\right)$ and $\mathrm{CD} 45^{+} \mathrm{CD} 11 \mathrm{c}^{+}$cells were significantly higher at 1 month after Mtb infection in the lungs of Mtb-infected Thra1 ${ }^{\mathrm{PV} /+}$ mice than in Mtb-infected WT control or uninfected Thra $1^{\mathrm{PV} /+}$ mice (Figure 7E and Supplemental Figures 15 and 16). Histological analysis revealed that the number of lesions throughout the lungs was significantly higher in the lungs of Mtb-infected Thra $1^{\mathrm{PV} /+}$ mice than in Mtb-infected WT mice or uninfected Thra ${ }^{\mathrm{PV} /+}$ mice (Figure 7, F and G).

\section{Discussion}

In the current study, we followed 839 healthy HHCs of TB patients for 2 years, obtained blood samples at 4-month intervals, and determined various factors correlated with the development of active TB in some of the HHCs. During the 24-month follow-up period, 17 HHCs developed active TB, and $14(82.4 \%)$ of 


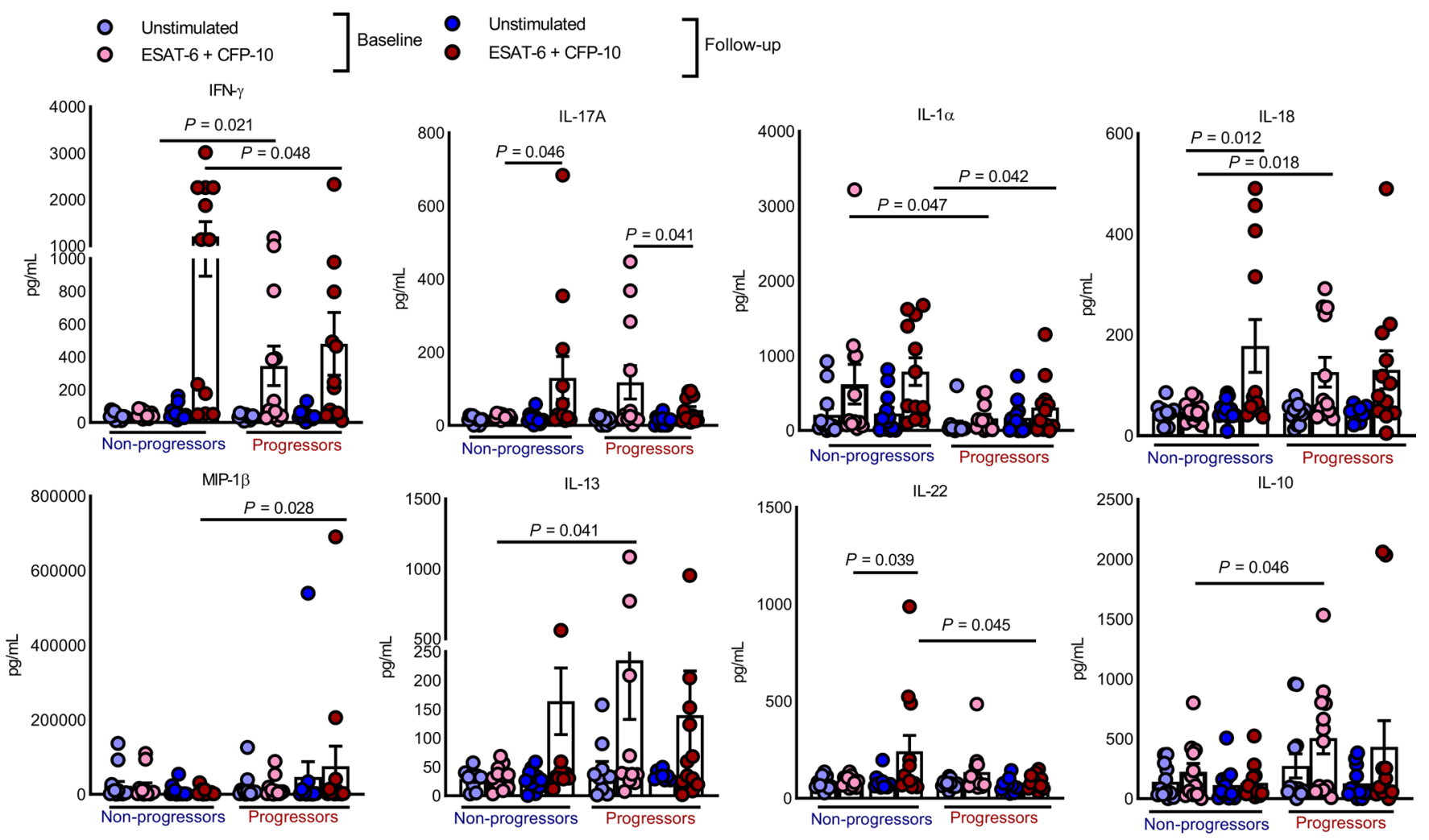

Figure 3. Cytokine and chemokine profiles of ESAT-6- and CFP-10-stimulated PBMCs. PBMCs from nonprogressors $(n=12)$ and progressors $(n=12)$ at baseline and follow-up were isolated and cultured with or without ESAT- 6 and CFP-10 $(10 \mu \mathrm{g} / \mathrm{mL}$ each), as described in Methods. After 96 hours, the culture supernatants were collected, and levels of various chemokines and cytokines were measured by multiplex ELISA. All the age-matched non-TB progressors were healthy, nonsmoking, and nonalcoholic and were without any immunosuppressive conditions at baseline and follow-up. Data from smoking and alcoholic progressors were not included. $P$ values were determined using 1-way ANOVA with Tukey's multiple-comparison test. The mean values, SEM, and $P$ values are shown.

these progressors were in the 15- to 30-year age group. This suggests that the development of active TB in HHCs is age-dependent (relative risk, 3.75). At baseline (the " 0 " time point, when all the individuals were healthy), levels of circulating thyroid hormone $\mathrm{T} 4$ were significantly lower in the progressors compared with age-matched nonprogressors. At baseline and follow-up (a blood sample was obtained when active TB developed), freshly isolated PBMCs of the progressors had more $\mathrm{CD} 16^{+} \mathrm{CD} 56^{+}$cells and Tregs than nonprogressors. At baseline, ESAT-6- and CFP-10-stimulated PBMCs of progressors produced more IFN- $\gamma$, IL-13, IL-10, and IL-18 and less IL-1 $\alpha$ compared with ESAT-6- and CFP-10-stimulated PBMCs of nonprogressors. In the presence of thyroid hormones $\mathrm{T} 3$ and $\mathrm{T} 4$, monocytes significantly inhibited Mtb growth in human MDMs in an IL-1 $\alpha$-dependent manner. We also found that Thra $1^{\mathrm{PV} /+}$ mice (with defective thyroid hormone signaling) were more susceptible to Mtb infection than WT mice. Our studies identified additional risk factors for the development of active TB in the healthy HHCs of TB patients.

The HHCs of TB patients in developing countries are at an increased risk of development of active TB (25). It is known that this risk is further enhanced by HIV infection, alcoholism, smoking, diabetes, and other immunosuppressive conditions (42-45). However, there is limited information about the risk factors that enhance the development of active TB in healthy HHCs (10-21). Age is a major factor associated with Mtb infection (46). Children are at an increased risk of TB infection and disease progression because of their immature immune system $(47,48)$. Similarly, the geriatric population represents a large reservoir of LTBI and is at high risk of developing active disease because of a weak immune system $(49,50)$. In the current study, we found that adolescents could be at risk for development of TB and that there was a correlation between decreased circulating thyroid hormone T4 levels in the progressors compared with age-matched nonprogressors. Hormones can directly affect the activity of immune cells and control inflammatory, autoimmune, or infectious immune responses $(51,52)$. The bidirectional communication between the neuroendocrine and immune systems is well established (53). Glucocorticoids can inhibit Th1 responses, whereas their natural antagonist DHEA is able to promote such responses (54). TB patients have altered thyroid hormone and 
A
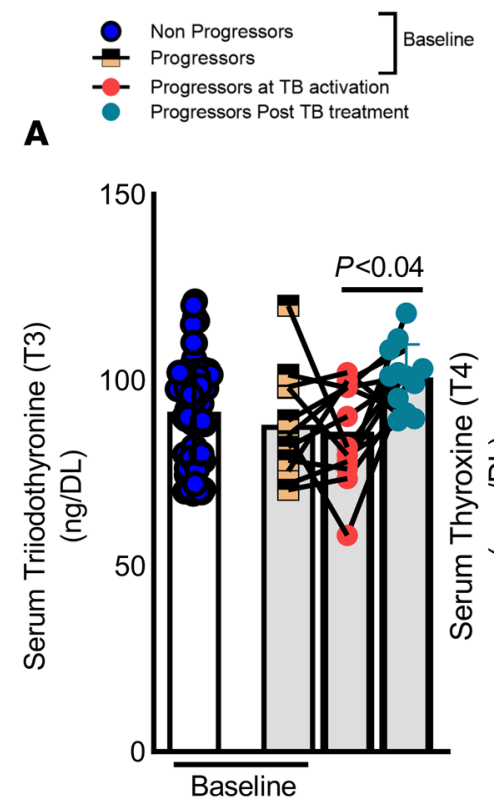

B

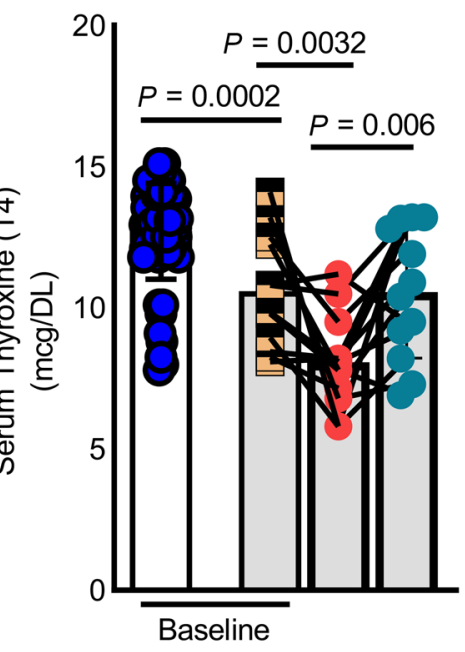

C

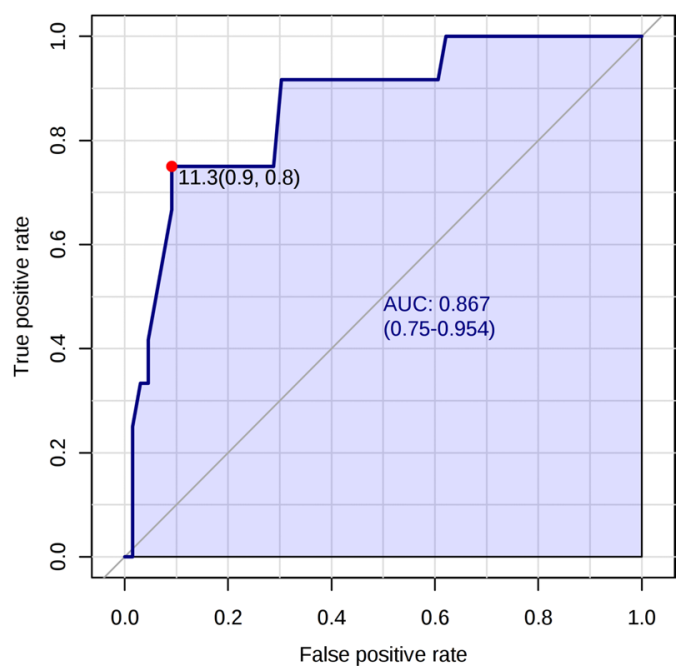

Figure 4. Hormone levels in the serum of HHCs of TB patients. Serum (A) T3 and (B) T4 hormone levels of the nonprogressors ( $n=67)$ and progressors $(n=12)$ at baseline and follow-up (at TB activation) were quantified by ELISA. All the age-matched nonprogressors were healthy, nonsmoking, and nonalcoholic and were without any immunosuppressive conditions at baseline or at follow-up. Data from smoking and alcoholic progressors were not included. $P$ values were derived using an unpaired, 2-tailed, independent $t$ test. Mean \pm SD and $P$ values are shown. (C) The receiver operating characteristic (ROC) curve for the hormone T4; plot shows the ROC curve for T4 hormone levels in nonprogressors $(n=67)$ and TB progressors $(n=12)$ at baseline. The ROC curve shows the true-positive rate (sensitivity) and false-positive rate (1-specificity) of the model prediction. The biomarker analysis program in MetaboAnalyst software was used to generate the ROC curves and calculate the AUC.

DHEA levels (55). In inbred rabbits, administration of $\mathrm{T} 3$ or T4 hormone markedly increased resistance to Mtb infection and hypothyroidism enhanced the susceptibility to Mtb infection (56). In the current study, HHCs who developed active TB disease during the follow-up period had a low level of serum T4 at baseline (Figure 4). TSH levels are regulated by T4 hormone through a negative feedback loop (57). However, our study found similar levels of TSH in nonprogressors and progressors at baseline (Supplemental Figure 4C). Our study demonstrated, to our knowledge, that decreased circulating T4 hormone levels at baseline can be a risk factor for young adult $\mathrm{HHCs}$ of $\mathrm{TB}$ patients.

At baseline, ESAT-6- and CFP-10-stimulated PBMCs of progressors produced less IL-1 $\alpha$ than the stimulated PBMCs of the nonprogressors (Figure 3). IL-1 $\alpha$-deficient mice are susceptible to infection with low-dose aerosolized Mtb (58). Furthermore, in vivo neutralization of IL-1 $\alpha$ renders mice highly susceptible to Mtb, and IL-1 $\alpha$ is essential for the establishment of host resistance to Mtb. Chronic Mtb infection in IL-1 $\alpha$ knockout mice causes large and diffuse inflammatory lesions in the lungs and the dispersed distribution of Mtb-infected cells (58). This suggests the important role of IL-1 $\alpha$-driven cell-cell crosstalk in coordinating protective granuloma formation or maintenance $(58,59)$. Our study demonstrated a correlation between decreased IL-1 $\alpha$ production at baseline and the development of active TB disease at follow-up.

We found that monocytes are the major cell population that express thyroid and glucocorticoid receptors. T3 and T4 but not cortisol and DHEA enhanced IL-1 $\alpha$ production by Mtb-infected macrophages and inhibited Mtb growth in an IL-1 $\alpha$-dependent manner. Thyroid receptor- $\alpha$ expression on macrophages modulates the inflammation caused by infection (60). Thyroid hormone-treated macrophages display the M1 phenotype and show improved capacity for phagocytosis (60). We have not determined the IL-1 $\alpha-$ dependent mechanisms that inhibit Mtb growth in macrophages. However, it is known that IL-1 signaling inhibits Mtb growth in a COX2-dependent manner by suppressing IFN-1 production (58). In the current study, we found that young HHCs with reduced circulating T4 levels develop active TB and found a mechanism by which the $\mathrm{T} 3$ and $\mathrm{T} 4$ hormones inhibited Mtb growth in an IL-1 $\alpha$-dependent manner.

In addition to the above findings, we also found higher numbers of $\mathrm{CD} 16^{+} \mathrm{CD} 56^{+}$cells and Tregs in fresh PBMCs; increased IFN- $\gamma$, IL-13, and IL-10 production; and reduced IL-1 $\alpha$ production by the ESAT6- and CFP-10-stimulated PBMCs of progressors compared with nonprogressors at baseline. NK cells are 

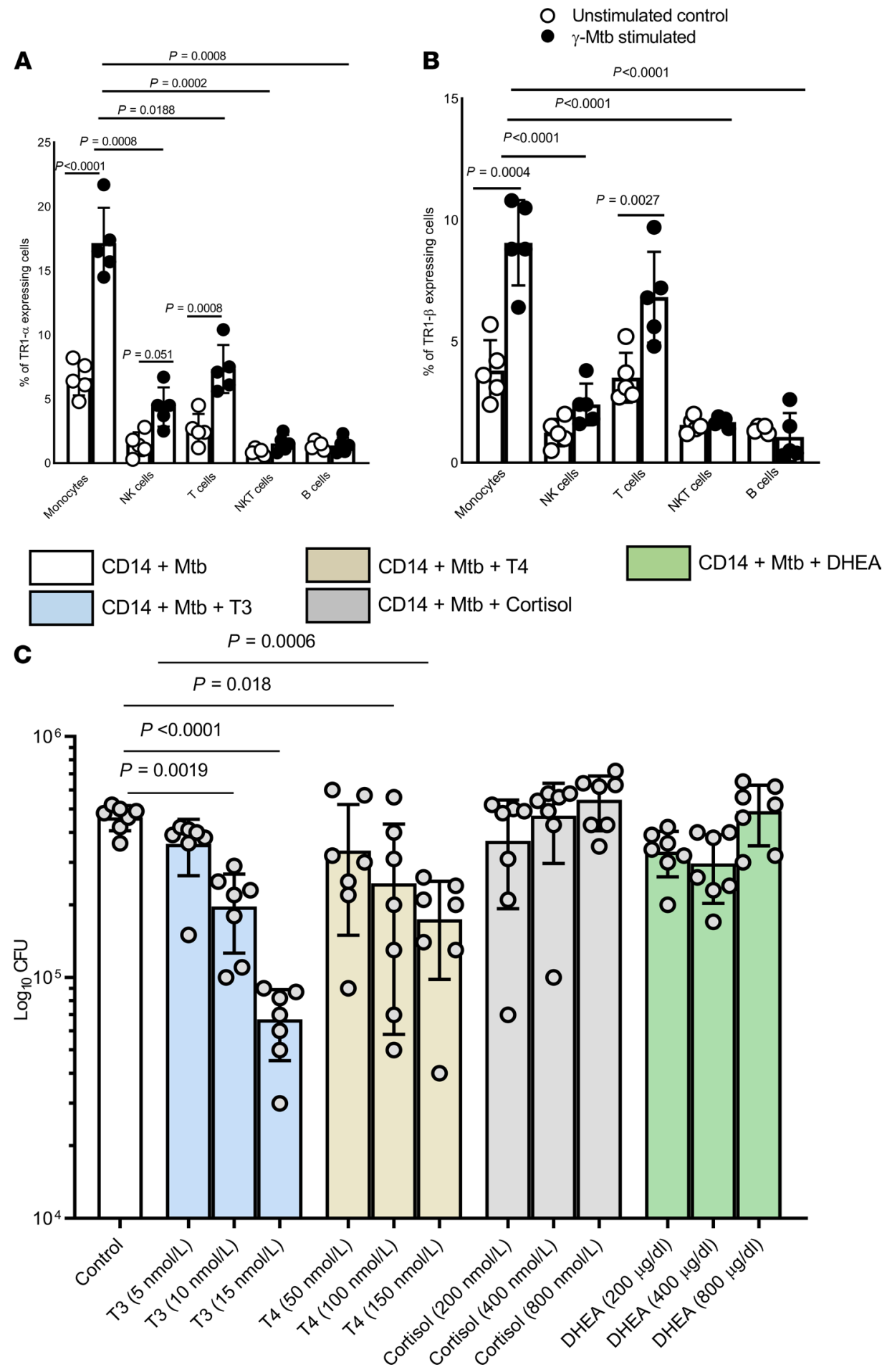

Figure 5. Thyroid hormones restrict Mtb growth in vitro. Freshly isolated PBMCs from 5 healthy individuals (age range: 18-30 years) were cultured with or without $\gamma$-irradiated Mtb H37Rv ( $\gamma$-Mtb, $10 \mu \mathrm{g} / \mathrm{mL}$ ) for 120 hours. (A) The percentages of TR1- $\alpha^{+}$and (B) TR1- $\beta^{+}$cells among monocytes, NK cells, T cells, NKT cells, and B cells were determined by flow cytometry. $P$ values were determined using 1-way ANOVA with Tukey's multiple-comparison test. The mean values, SD, and $P$ values are shown. (C) Mtb growth in monocyte-derived macrophages (MDMs). The MDMs from healthy individuals $(n=$ 7) were infected with H37Rv at an MOI of 2.5 as described in Methods. Some of the infected MDMs were cultured in the presence of T3 $(5,10,15 \mathrm{nmol} / \mathrm{L})$, T4 (50, 100, $150 \mathrm{nmol} / \mathrm{L})$, DHEA (200, 400, $800 \mu \mathrm{g} / \mathrm{dl})$, or cortisol $(200,400,800 \mathrm{nmol} / \mathrm{L})$ for 5 days. The supernatant was aspirated, and the MDMs were lysed. Supernatant was centrifuged to pellet the bacteria, and the pellets were added to the cell lysates. The bacterial suspensions were ultrasonically dispersed, serially diluted, and plated in triplicate on $7 \mathrm{H} 10$ agar. The number of resultant colonies was counted after 3 weeks. The $P$ values were derived using an unpaired, 2-tailed, independent $t$ test. The mean values and SD are shown for the number of CFUs per well. 
an important component of the innate immune response and kill Mtb-infected cells $(61,62)$. CD16 (Fc receptor $\gamma \mathrm{RIII}$ ) is a mediator of antibody-dependent cell-mediated cytotoxicity and is expressed by a subpopulation of NK cells (63). Antibody recognition by CD16 results in the activation of NK cells, release of cytolytic granules, and the killing of target cells (63). In the current study, we found that CD16-expressing NK cell numbers were high in the progressors at baseline compared with nonprogressors, and this number was reduced when HHCs developed active TB disease. We have not determined the function of CD16 NK cells in progressors and nonprogressors, but our study results are in accordance with those of a recent cohort study that found lower numbers of peripheral blood NK cells in patients with active TB, whereas individuals with LTBI had a normal NK cell number. Recent studies in nonhuman primates demonstrated that $\mathrm{CD} 27^{+} \mathrm{NK}$ cells accumulate more in the lung of LTBI-positive macaques compared with macaques with active TB (64). This NK cell population was also detected in circulation of LTBI-positive individuals and expanded in response to $\mathrm{Mtb}$ antigens (33).

At baseline and follow-up (at the time of the development of active disease), we found higher numbers of Tregs in the PBMCs of the progressors compared with those in the PBMCs of the nonprogressors. Activated $\mathrm{CD}^{+} \mathrm{T}$ cells can transiently express CD25 and FoxP3 (65), and we have not measured the suppressive function of $\mathrm{CD}^{+} \mathrm{CD} 25^{+} \mathrm{FoxP}^{+}$cells. It is possible that increased numbers of $\mathrm{CD} 4^{+} \mathrm{CD} 25^{+} \mathrm{FoxP} 3^{+}$cells in the $\mathrm{PBMCs}$ of the progressors may be due to increased activated CD4 ${ }^{+}$cells. ESAT-6- and CFP-10-stimulated PBMCs of progressors produced more IL-10 than the stimulated PBMCs of nonprogressors at baseline and at follow-up. Tregs produce IL-10 and play an important role in modulating Mtb-specific immune responses (66). Tregs in lymph nodes reduce the frequency of Mtb-responsive IFN- $\gamma$-producing $\mathrm{CD} 4^{+}$and $\mathrm{CD} 8^{+} \mathrm{T}$ cells, and there is an association between the number of Tregs and the severity of Mtb infection $(31,67)$. The higher preinfection frequencies of Treg cells in Mtb-infected cynomolgus macaques was associated with a higher likelihood of latent TB (68). Our study suggests that the increased numbers of Tregs along with increases in other markers at baseline in young HHCs of TB patients can serve as a biomarker for the future development of active TB.

We found that the Mtb antigen-stimulated PBMCs of the progressors produced more IFN- $\gamma$ and IL-13 than the stimulated PBMCs of the nonprogressors at baseline. In contrast, the ESAT-6- and CFP-10-stimulated PBMCs of progressors produced less IFN- $\gamma$, IL-17, and IL-1 $\alpha$ compared with the stimulated PBMCs of the nonprogressors at follow-up (when the progressors developed active TB). The essential protective role of these cytokines during Mtb infection is well known (69). The higher numbers of $\mathrm{CD}^{2} 6^{+} \mathrm{CD} 16^{+}$cells and higher levels of protective cytokine production at baseline in the progressors suggest that strong host defense mechanisms are in place to protect the progressors during the early stages of infection, but the failure of these mechanisms leads to the development of active TB disease. This failure was demonstrated by reduced numbers of $\mathrm{CD} 56^{+} \mathrm{CD} 16^{+}$cells and the production of associated cytokines in progressors.

Similar to the above findings in humans, Mtb-infected Thra $1^{\mathrm{PV} /+}$ mice demonstrated defective effector cytokine (IL-1 $\alpha$ and IFN- $\gamma$ ) and chemokine (IP-10, MIP-1 $\alpha$, MIP-1 $\beta$, and MCP-3) production, a higher number of Tregs, a higher bacterial burden in the lungs, and higher mortality than Mtb-infected WT mice (45.4\% of the mice died within 65 days, Figure 7). Thyroid hormone signaling can affect immune cell functions, such as chemotaxis, phagocytosis, ROS generation, cytokine production, and metabolism (70). Given that T4 and T3 play pleiotropic roles in metabolism, our findings with Thra $1^{\mathrm{PV} /+}$ mice may not be specific for Mtb infection and it is possible that Thra $1^{\mathrm{PV} /+}$ mice may be susceptible to other infections. Our human and mouse findings suggest that thyroid hormone signaling is essential for the generation of optimal immune responses during Mtb infection.

In summary, we followed a large number of healthy HHCs of TB patients, obtained blood samples at regular intervals, and identified the risk factors that enhanced the development of active TB. Our findings suggest that young HHCs of TB patients with increased numbers of $\mathrm{CD} 16^{+} \mathrm{CD} 56^{+}$cells and Tregs as well as decreased production of T4 hormone and IL-1 $\alpha$ at baseline are at an increased risk of developing active TB disease.

\section{Methods}

\section{Human studies}

Cohort description. Over 4 years, a total of 839 household contacts were enrolled in the study. The following were used as the enrollment criteria.

Index patients were newly diagnosed, sputum-positive pulmonary TB patients attending the Designated Microscopy Centers under TB clinics at Bhagwan Mahavir Medical Research Centre (BMMRC) and Blue Peter 
A

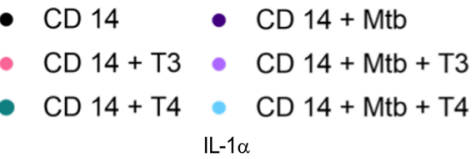

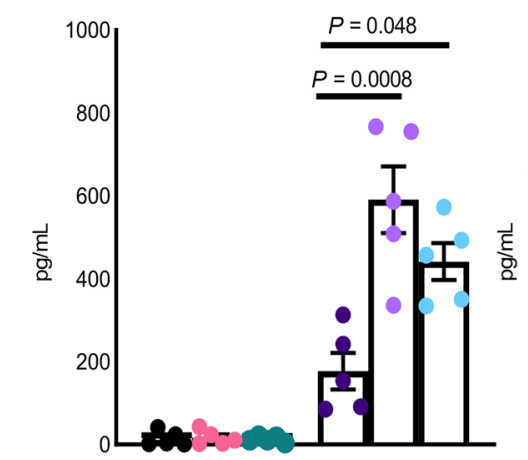

TNF- $\alpha$

CCL3/MIP-1 $\alpha$

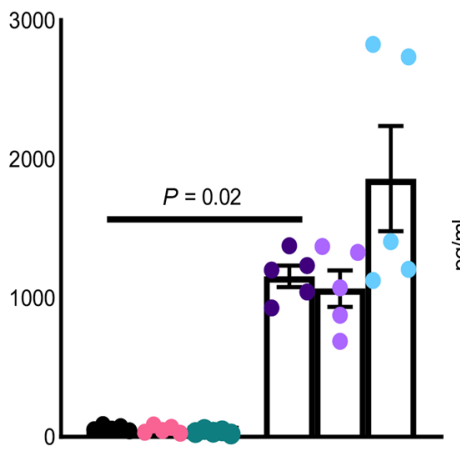

B

$\mathrm{TR}-\alpha 1 / \beta 1$

$\mathrm{IL}-1 \mathrm{a}$

DAPI
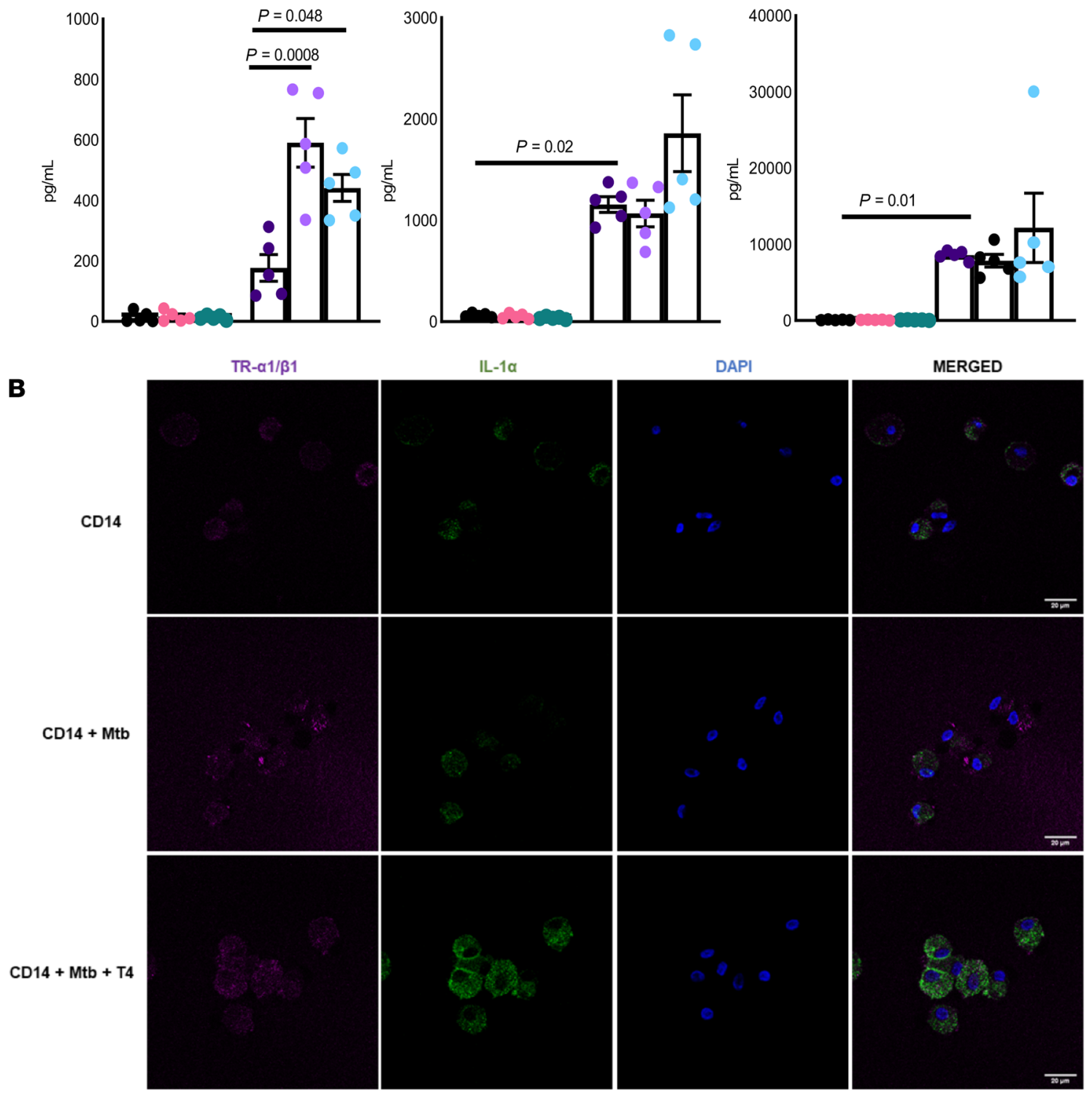

C

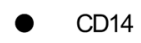

$$
\text { - CD14+Mitb }
$$

- $\mathrm{CD} 14+\mathrm{Mtb}+\mathrm{T} 4$
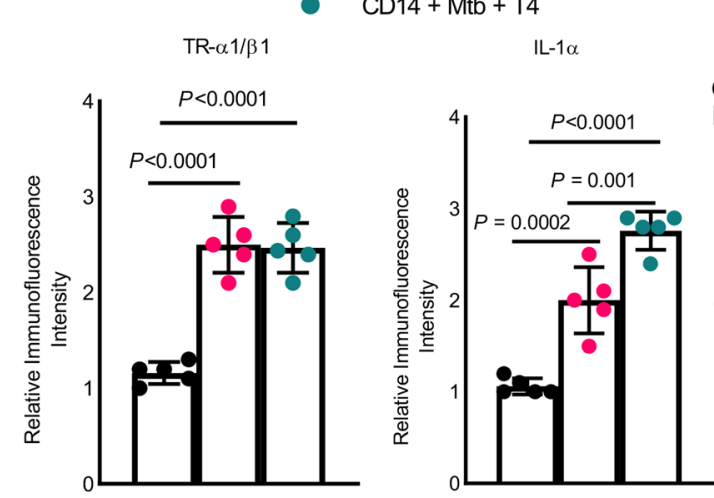

D

- $\mathrm{CD} 14+\mathrm{M}$ tb

- $\mathrm{CD} 14+\mathrm{Mtb}+\mathrm{T} 4$

O CD14 + Mtb + T4 + Isotype Antibody

- $\mathrm{CD} 14+\mathrm{Mtb}+\mathrm{T} 4+$ Anti-IL-1 $\alpha$ Antibody

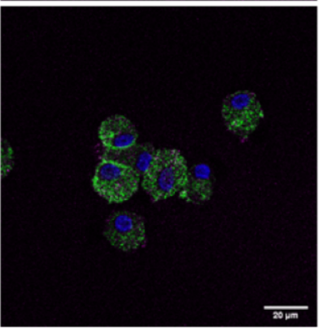
${ }^{10^{6}} \mathbf{7}^{P<0.0001}$

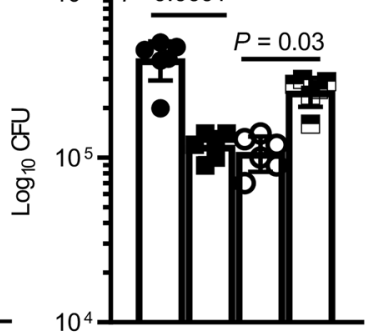

E

- $\mathrm{CD} 14+\mathrm{Mtb}$

- $\mathrm{CD} 14+\mathrm{Mtb}+\mathrm{rlL}-1 \alpha$

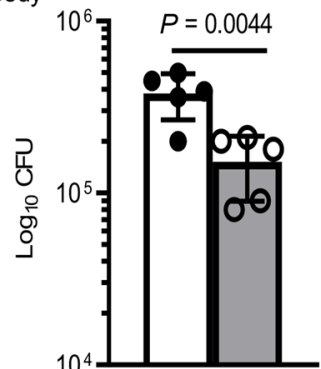


Figure 6. Thyroid hormones induce IL-1 $\alpha$ production by Mtb-infected monocytes/monocyte-derived macrophages. Freshly isolated monocytes/monocytederived macrophages (MDMs) from 18- to 30-year-old donors $(n=5)$ were infected with H37Rv at an MOI of 2.5, as described in Methods. Some of the infected MDMs were cultured in the presence of $\mathrm{T} 3(15 \mathrm{nmol} / \mathrm{L})$ or T4 $(150 \mathrm{nmol} / \mathrm{L})$ for 5 days. (A) The supernatants were aspirated, and cytokine and chemokine production were measured by multiplex ELISA. $P$ values were derived using 1-way ANOVA with Tukey's multiple-comparison test. The mean values, SD, and $P$ values are shown. (B) The Mtb-infected MDMs in the presence or absence of T4 $(150 \mathrm{nmol} / \mathrm{L})$ were examined for TR- $\alpha 1 / \beta 1$ expression and IL- $1 \alpha$ production by confocal microscopy. The images were taken at $63 \times$ magnification with oil immersion. Scale bar: $20 \mu \mathrm{m}$. (C) The respective immunofluorescence intensities are shown $(n=5)$. $P$ values were derived using 1-way ANOVA with Tukey's multiple-comparison test. Mean values, $P$ values, and SD are shown. (D) Freshly prepared MDMs from 18- to 30-year-old donors $(n=6)$ were infected with H37Rv at an MOI of 2.5, as described in Methods. The infected MDMs were cultured in the presence of $\mathrm{T} 4(150 \mathrm{nmol} / \mathrm{L})$, and the anti-IL-1 $\alpha(10 \mu \mathrm{g} / \mathrm{mL})$ antibody was added to some wells. The supernatant was aspirated; MDMs were lysed and plated on 7H10 agar. The number of colonies was counted after 3 weeks. The mean \pm SD and $P$ values are shown. The $P$ values were determined using 1-way ANOVA with Tukey's multiple-comparison test. (E) The MDMs $(n=5)$ were infected with H37Rv and cultured in the presence or absence of recombinant IL-1 $\alpha(10 \mathrm{ng} / \mathrm{mL})$; the number of bacterial colonies was determined. The $P$ values were derived using an unpaired, 2-tailed, independent $t$ test. Mean values and SD are shown.

Public Health \& Research Centre (BPHRC) in Hyderabad, India. Active TB diagnosis was performed by following the Revised National Tuberculosis Control Programme (RNTCP) (now National Tuberculosis Elimination Programme, NTEP) guidelines.

Household members residing in the same house as the index case for a minimum of 3 months prior to the date of diagnosis of TB were identified. These individuals had shared at least 5 meals per week with the index case and had no past history of TB or ATT. The demographic details of the HHCs, including age, sex, history of Bacillus-Calmette-Guerin (BCG) vaccination, history of pulmonary TB, and smoking and drinking profiles, were collected and are shown in Table 1.

The exclusion criteria were HHCs having HIV, diabetes, autoimmune diseases, or any other immunosuppressive condition; children younger than 15 years were excluded. If participants developed comorbid conditions and started using tobacco (chewing tobacco or smoking) or alcohol (more than 2 drinks per week) during the study, their data were excluded from the final analysis.

Initial screening of the participants. Prior to enrollment in the study, HHCs were screened to ensure that they meet the eligibility criteria outlined above. Screening evaluations included eligibility assessment, informed consent, detailed medical history, and exposure assessment to the index case. Local laboratory evaluations to rule out HIV, diabetes, and any other chronic conditions were performed. After enrollment, baseline visits were scheduled and blood samples were collected. Demographics and data on smoking and alcohol usage were collected. Physical examination was done and participants' height and weight were noted. Blood samples were used to perform immune assays and hematological analysis. Participants were categorized as having LTBI by an in-house assay as outlined in the section below.

Latent TB in the study cohort. HHCs were categorized as LTBI-positive or LTBI-negative using an in-house interferon gamma release assay (IGRA) (Supplemental Data Set 1) (71). The test was performed every 4 months irrespective of baseline IGRA result. The HHCs with symptoms consistent with TB were evaluated by chest radiography and clinical evaluation. A complete blood panel was performed to study the hematological correlates of the progression to TB as outlined in Supplemental Figure 1.

Follow-up of HHCs. All HHCs were evaluated every 4 months for 2 years. At each visit, they were evaluated for TB, and $20 \mathrm{~mL}$ of blood was collected. Blood samples were used to perform immune assays and hematological analysis. An in-house assay was performed to identify LTBI converters and nonconverters (71). All participants with symptoms of possible TB were referred for clinical evaluation and treatment.

Outcome determination. The outcomes of the study were as follows: 1) completion of the 2-year follow-up; 2) development of TB in some HHCs; and/or 3) withdrawal of the participants from the study.

Progressors were classified as those with signs and symptoms consistent with active TB and those with 1) clinical specimens that showed acid-fast bacilli in the test smear or Mtb in culture or 2) chest radiographic changes consistent with TB and a clinical response to ATT. The nonprogressors were individuals who completed their follow-up without development of TB.

$T B$ treatment in active TB progressors. Seventeen of the HHCs progressed to active TB during the follow-up. These HHCs were referred to the RNTCP (now NTEP) centers for treatment. All the TB progressors were screened for rifampicin resistance following RNTCP guidelines, and no drug resistance was observed in any of them. Standard fixed-dose combination (FDC) first-line ATT was administered to the patients according to their weight bands. Treatment was administered daily for a minimum of 6 months with 4 standard FDC drugs in the intensive phase and 3 drugs during the continuation phase. Although TB activation was an off-study criterion in this study, samples were collected from progressors after TB treatment for hormone assays. 
A

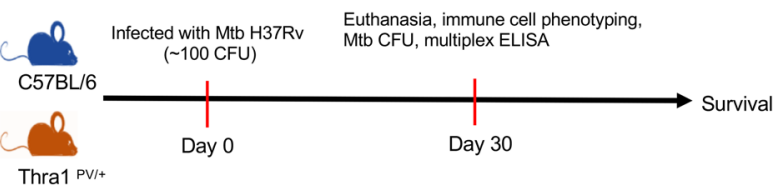

WT control

- Thra $1^{\mathrm{PV} / \mathrm{+}}$
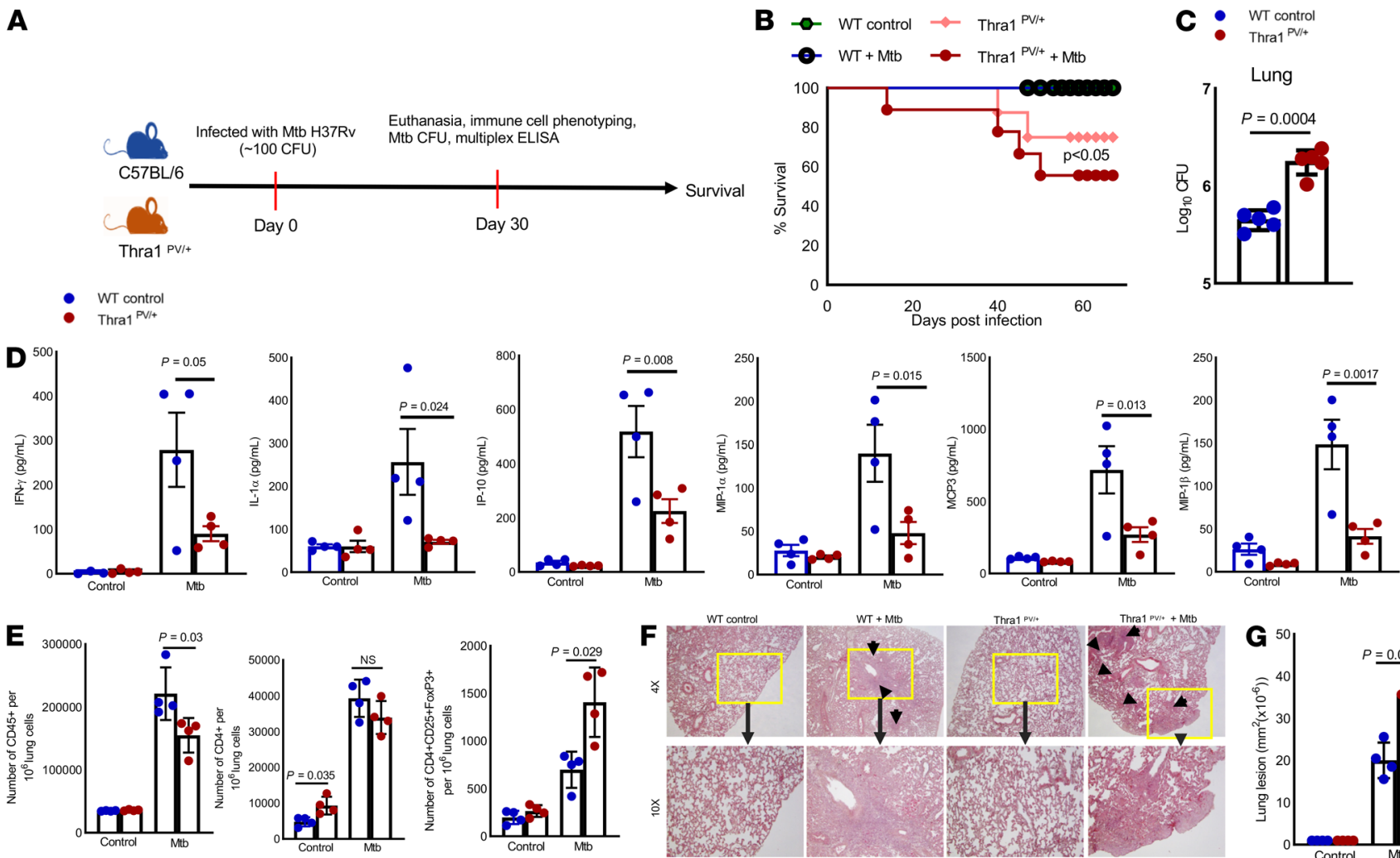

Figure 7. Thra ${ }^{\mathrm{PV} /+}$ mice are susceptible to Mtb infection. Six- to 8 -week-old WT and Thra1 ${ }^{\mathrm{PV} /+}$ mice (both C57BL/6 background) (10 mice per group) were infected with approximately 100 CFUs of Mtb H37Rv by aerosol inhalation. (A) A schematic representation of the experiment is shown. (B) The $P$ value for percentage survival was calculated using the log-rank test. The Kaplan-Meier survival curves of mice are shown. Data were pooled from 2 independent experiments ( $n=5$ mice in 2 experiments). (C) One month after infection, the bacterial burden in terms of CFUs in the lungs ( $n=5$ per group) was measured. Mean \pm SD and $P$ values are shown. The $P$ values were derived using 1-way ANOVA with Tukey's multiple-comparison test. (D) At 1 month after infection, lung homogenates from uninfected control and Thra $1^{\mathrm{PV} /+}$ mice and Mtb-infected control and Thra1 ${ }^{\mathrm{P} / /+}$ mice $(n=4)$ were collected, and cytokine and chemokine levels were measured with multiplex ELISA. (E) The numbers of $\mathrm{CD} 45^{+}, \mathrm{CD}^{+}$, and Tregs (CD4+CD25+FoxP3 $)$in various immune cell populations were measured by flow cytometry. Mean $\pm \mathrm{SD}$ and $P$ values are shown. $P$ values were derived using 1-way ANOVA with Tukey's multiple-comparison test. (F) At 1 month after infection, lungs from uninfected control and Thra ${ }^{\mathrm{PV} /+}$ mice and those from Mtb-infected control and Mtb-infected Thra1 ${ }^{\mathrm{PV} /+}$ mice were isolated and formalin-fixed. Paraffin-embedded tissue sections were prepared, and H\&E staining was performed. Total original magnification power $=10$ ocular $\times 4$ objective $=40$ and 10 ocular $\times 10$ objective $=100$.(G) Lung lesions were compared between groups. Data are representative of 2 independent experiments ( $n=4$ mice per group). $P$ values were derived using 1-way ANOVA with Tukey's multiple-comparison test. Data are expressed as the mean \pm SD and $P$ values are shown.

Determination of LTBI in the HHCs. LTBI in the HHCs was determined according to our previously published protocols (71). A total of $2 \times 10^{6}$ human PBMCs were stimulated with and without $10 \mu \mathrm{g} / \mathrm{mL} \mathrm{CFP-10} \mathrm{and}$ ESAT- 6 antigens and incubated at $37^{\circ} \mathrm{C}$ for 96 hours. IFN- $\gamma$ released by PBMCs was measured with a sandwich ELISA using a commercial human IFN- $\gamma$ kit (eBioscience Inc.) by following the manufacturer's instructions. The IFN- $\gamma$ concentration was calculated using MPM software version 6.1. HHCs were categorized as either LTBI-negative or LTBI-positive depending on the IFN- $\gamma$ value.

Complete blood count analysis. Whole blood collected in EDTA was subjected to complete blood count analysis by using an automated cell counter (Mindray Biochemistry Analyzer, Golden Harvest Industries). A total of $200 \mu \mathrm{L}$ of the whole blood collected in an EDTA tube was used for the complete blood count analysis. The WBC, RBC, and platelet counts were determined by impedance method, and hemoglobin levels were determined by colorimetry.

Antibodies and other reagents. PE anti-CD16 (catalog 555407), PerCP anti-CD14 (catalog 340585), and APC anti-CD56 (catalog 555518) were used to identify monocytes and NK cells. FITC anti-CCR7 (catalog 561271), PE anti-CD27 (catalog 555441), PerCP anti-CD3 (catalog 555332), and APC anti-CD56 (catalog 555518) were used to identify the T cell and NK cell populations. FITC anti-CD4 (catalog 555346), PE anti-Foxp3 (catalog 560046), and APC anti-CD25 (catalog 555434) were used to label Tregs (all antibodies used were obtained from $\mathrm{BD}$ Biosciences). To determine the expression of thyroid hormone receptors, we used Alexa Fluor 488 anti-thyroid hormone receptor $\beta$ and FITC-thyroid hormone receptor alpha (Bioss Antibodies) and performed intra- 
cellular staining using an intracellular staining kit (BioLegend). In addition, we used PE anti-human GPR83 (BioLegend) to determine the expression of glucocorticoid receptors.

Isolation and culture of PBMCS. PBMCs were isolated by density gradient centrifugation using FicollHypaque. Whole blood diluted with RPMI was layered over an equal volume of Ficoll and centrifuged for 30-40 minutes at 2000 RPM without braking. PBMCs were washed twice and counted by trypan blue staining. Freshly isolated PBMCs were cultured in 24-well plates at $1 \times 10^{6}$ cells/well in RPMI 1640 containing $1 \%$ penicillin/streptomycin (Sigma-Aldrich), L-glutamine, and 10\% heat-inactivated human serum with or without CFP-10 + ESAT-6 $(10 \mu \mathrm{g} / \mathrm{mL})$ at $37^{\circ} \mathrm{C}$ in a humidified atmosphere with $5 \% \mathrm{CO}_{2}$. After 96 hours, cell-free culture supernatants were collected, aliquoted, and stored at $-70^{\circ} \mathrm{C}$ until the cytokine concentrations were measured by ELISA according to the manufacturer's guidelines.

Antigens for stimulation assays. For stimulation of PBMCs, we used ESAT-6 and CFP-10 peptide pools (BEI Resources), consisting of 21 and 22 peptides covering the entire $6 \mathrm{kDa}$ ESAT- 6 and $10 \mathrm{kDa}$ CFP-10 protein sequence, respectively. A total of $1 \times 10^{6}$ human PBMCs were stimulated with and without $10 \mu \mathrm{g} /$ $\mathrm{mL}$ CFP-10 and ESAT- 6 antigen and incubated at $37^{\circ} \mathrm{C}$ for 96 hours. For some experiments, freshly isolated PBMCs were cultured with or without $\gamma$-Mtb H37Rv (BEI Resources) $(10 \mu \mathrm{g} / \mathrm{mL})$ for 120 hours.

Flow cytometry. Briefly, a total of $1 \times 10^{6}$ PBMCs were stained for 3 cell subsets. T cells, monocytes, and the NK cell subsets were surface stained with respective antibodies and incubated in the dark for 30 minutes before being analyzed by flow cytometry. To determine the FoxP3 population, we used an intracellular staining kit from BioLegend. For surface staining, CD4 and CD25 were added to the cells before they were permeabilized. Anti-FoxP3 antibody was then added to the cells resuspended in staining buffer. After incubation, the cells were washed twice and fixed in 1\% paraformaldehyde before acquisition on a FACSCalibur (BD Biosciences).

Gating strategies. $\mathrm{CD}^{+}$cells were identified after gating on the whole lymphocyte population. Tregs were gated as $\mathrm{CD}^{+}{ }^{+} \mathrm{CD} 25^{+} \mathrm{FoxP}^{+}$cells. CD16-expressing $\mathrm{CD}^{+} 6^{+}$cells were identified as double-positive $\mathrm{CD} 16^{+} \mathrm{CD} 56^{+}$ cells. CD16-expressing monocytes were identified as $\mathrm{CD} 14^{+} \mathrm{CD} 16^{+}$cells.

Measurement of T3, T4, DHEA, and cortisol. T3, T4, DHEA, and cortisol concentrations in the serum were measured by ELISA by following the manufacturer's instructions (T3 and T4, Meril Diagnostics) (DHEA and cortisone, Diagnostics Biochem Canada). Briefly, $25 \mu \mathrm{L}$ of standards (positive controls provided by the manufacturer for the purpose of assay) or patient sera were added to the respective precoated wells. Fifty microliters of biotin and $100 \mu \mathrm{L}$ of the enzyme conjugate were added to all the wells. The samples were incubated for 60 minutes at room temperature (RT). The wells were washed, and $100 \mu \mathrm{L}$ of the substrate was added. The stop solution was added after 15-20 minutes of incubation in the dark. The absorbance was read at $450 \mathrm{~nm}$. The samples were run in batches, and assays were performed by commercial vendors who were blinded to the specimen status.

Measurement of cytokine production. ESAT-6- and CFP-10-stimulated PBMCs as well as the T3-, T4-, DHEA-, and cortisol-treated $\mathrm{H} 37 \mathrm{Rv}$-infected MDM culture supernatants were stored at $-70^{\circ} \mathrm{C}$ until the cytokine concentrations were measured. In culture supernatants, the following 34 cytokines and chemokines were measured using a multiplex ELISA kit per the manufacturer's instructions (34-Plex Human ProcartaPlex Panel 1A, Thermo Fisher Scientific): Eotaxin/CCL11; GM-CSF; GRO- $\alpha / C X C L 1 ;$ IFN- $\alpha$; IFN- $\gamma$; IL-1 $\beta$; IL-1 $\alpha$; IL-1RA; IL-2; IL-4; IL-5; IL-6; IL-7; IL-8/CXCL8; IL-9; IL-10; IL-12 p70; IL-13; IL-15; IL-17A; IL-18; IL-21;

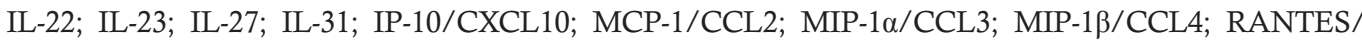
CCL5; SDF1- $\alpha /$ CXCL12; TNF- $\alpha$; and TNF- $\beta /$ LTA. The assay was run in batches by an independent reader blinded to the specimen status.

Determination of Mtb H37Rv growth in MDMs. Monocytes were isolated from the PBMCs of healthy donors by magnetic beads conjugated to anti-CD14 (Miltenyi Biotec). CD14 ${ }^{+}$monocytes $\left(0.5 \times 10^{6}\right.$ cells/well) were plated in 24-well plates in $1 \mathrm{~mL}$ of antibiotic-free macrophage serum-free medium (SFM) (Gibco) and incubated at $37^{\circ} \mathrm{C}$ in a humidified atmosphere in $5 \% \mathrm{CO}_{2}$ for up to 6 days. The culture medium was renewed every day, and the cells were monitored morphologically for differentiation. After 6 days (after differentiation of the monocytes into macrophages), the cells were washed and infected with Mtb H37Rv at an MOI of 2.5 (1 MDM to $2.5 \mathrm{Mtb}$ ), incubated at $37^{\circ} \mathrm{C}$ for 2 hours, washed to remove the extracellular bacilli, and cultured in macrophage-SFM (Gibco). T3 (5, 10, and $15 \mathrm{nmol} / \mathrm{L}), \mathrm{T} 4$ (50, 100, and $150 \mathrm{nmol} / \mathrm{L})$, DHEA (200, 400, and 800 $\mu \mathrm{g} / \mathrm{dl})$, and cortisol $(200,400$, and $800 \mathrm{nmol} / \mathrm{L})$ were added to some of the wells. Anti-IL-1 $\alpha(10 \mathrm{ng} / \mathrm{mL})$ was added to some of the wells. The cells were cultured for 120 hours. The supernatant was aspirated, and MDMs were lysed. The supernatant was centrifuged to pellet the bacteria, and the pellets were added to the cell lysates. 
The bacterial suspensions were ultrasonically dispersed, serially diluted, and plated in triplicate on 7H10 agar. The number of colonies was counted after 3 weeks.

Confocal microscopy. Confocal microscopy was performed to determine IL- $1 \alpha$ and TR- $1 \alpha / \beta$ expression on control or Mtb-infected monocytes in the presence or absence of T4 hormone. The CD14 ${ }^{+}$monocytes $\left(0.5 \times 10^{6}\right.$ cells/well) were plated in 24 -well plates in $1 \mathrm{~mL}$ of antibiotic-free macrophage-SFM (Gibco) and incubated at $37^{\circ} \mathrm{C}$ in a humidified atmosphere with $5 \% \mathrm{CO}_{2}$. After 2 hours, the cells were washed and infected with Mtb H37Rv at an MOI of 2.5 (1 MDM to $2.5 \mathrm{Mtb}$ ), incubated at $37^{\circ} \mathrm{C}$ for 2 hours, washed to remove the extracellular bacilli, and cultured in macrophage-SFM. T4 hormone was added to a few wells at a concentration of $150 \mathrm{nmol} / \mathrm{L}$. For immunostaining, the cells were first washed briefly with PBS and fixed with 4\% paraformaldehyde for 15-20 minutes at RT. The samples were then washed 3 times for 5 minutes with PBST. Fixed cells were then incubated with $0.025 \%$ Triton X-100 in PBS (PBST) for 10 minutes and subjected to 3 subsequent washes in PBST. Nonspecific binding was blocked by incubating the samples with 5\% BSA in PBST for 1 hour and then washing 2 times for 5 minutes in PBST. The cells were then incubated at $4^{\circ} \mathrm{C}$ overnight in PBST with appropriate dilutions of the primary antibodies IL-10 (1:250) and TR-1 $\alpha / \beta$ (1:250), and subsequently the cells were washed thoroughly for 3 times for 5 minutes in PBST. Next, the cells were stained with their respective secondary antibodies at 1:1000 dilutions (v/v), washed again with PBST for 3 times for 5 minutes, and mounted using FluoroShield mounting medium with DAPI. The mounted slides were then examined under a Zeiss LSM 510 meta laser scanning confocal microscope. ZEN 2009 software (Zeiss) was used for 3-5 image acquisitions per sample; then, the images were processed and uniformly quantified for each experiment using ImageJ software (NIH). Representative images are shown from 5 independent experiments.

Western blot. $\mathrm{CD}_{14}^{+}$monocytes were isolated from PBMCs of healthy volunteers and cultured at $1 \times 10^{6}$ cells in 12-well plates to obtain MDMs for 2-3 days. MDMs were infected with Mtb H37Rv, and protein lysates were collected after 72 hours of infection using M-PER (mammalian protein extraction reagent) with protease and phosphatase inhibitor cocktail (Thermo Fisher Scientific). Total protein concentrations were estimated using the bicinchoninic acid method, and $15 \mu \mathrm{g}$ of protein samples were used to perform Western blotting. Resolved protein gels were electroblotted onto a PVDF membrane and blocked with $5 \%$ nonfat dry milk in TBST buffer for 1 hour at RT. Then, the membranes were probed with Rb-DIO1 (Invitrogen, PA5-100139; 1:1000), goat-DIO2 (Novus, NBP1-00178; 1:1000), Rb-DIO3 (Novus, NBP1-05767; 1:1000), and Rb-GAPDH (Cell Signaling Technology, 2118; 1:1000) overnight at $4^{\circ} \mathrm{C}$ and followed by anti-rabbit (Cell Signaling Technology, 7074; 1:10000) or anti-goat (Santa Cruz Biotechnology, sc-2354; 1:3000) HRP-linked secondary antibody for 1 hour at RT. Protein bands were detected using the enhanced chemiluminescence method using Bio-Rad ChemiDoc imaging system.

Statistical analysis used for human studies. Prism 7 software (GraphPad) was used for statistical analyses. Descriptive analyses were performed for all relevant variables prior to their inclusion in analyses. Frequency counts and percentages were obtained for interval and ordinal categorical data. For continuous variables, the central tendency (mean, mode, and median) and dispersion (range, variance, standard deviation, standard error, and coefficient of variation) were calculated. Possible outliers and/or influential observations were identified, and their validity was double-checked using available records. $P$ values that were less than 0.05 were considered statistically significant. The results are expressed as the mean $\pm \mathrm{SD}$. For data that were normally distributed, comparisons between groups were assessed with ANOVA and 1-way repeated-measures ANOVA. When the overall ANOVA $P$ values were statistically significant and post hoc tests were performed ( $t$ tests), $P$ values were adjusted for multiple comparisons using Tukey's multiple-comparison test. For data that were not normally distributed, Wilcoxon's rank-sum test was used. The biomarker analysis program was used in MetaboAnalyst software to generate the ROC curves, and the AUC was determined for various hormones to predict the true TB progressors at baseline.

Human study approval. From June 2014 through June 2018, we enrolled 839 HHCs (Figure 1) aged 15-73 years after obtaining written informed consent. The study was approved by the institutional ethics committees from BMMRC and BPHRC.

\section{Mouse studies}

Animals. Specific pathogen-free 4- to 8-week-old male and female WT C57BL/6 mice were obtained from The Jackson Laboratory, and Thra1 ${ }^{\mathrm{PV} /+}$ (C57BL/6 background) mice were provided in-house. Thra1 ${ }^{\mathrm{PV} /+}$ founder mice were back-crossed into the C57BL/ 6 background and maintained at the animal facility in the 
University of Texas Health Science Center at Tyler. Thra ${ }^{\mathrm{PV} /+}$ mice and littermates were genotyped (primers are listed in Supplemental Table 4) using the REDExtract-N-Amp Tissue PCR kit (MilliporeSigma) to confirm the presence of the approximately 586-bp PCR product.

Aerosol infection of mice with Mtb H37Rv. Before infecting the mice with Mtb H37Rv, bacteria were grown in liquid medium until the mid-log phase was reached and then frozen in aliquots at $-70^{\circ} \mathrm{C}$. Bacterial counts were determined by plating on 7H10 agar supplemented with oleic albumin dextrose catalase (OADC). For infection, bacterial stocks were diluted in $10 \mathrm{~mL}$ of normal saline $\left(0.5 \times 10^{6} \mathrm{CFU} / \mathrm{mL}, 1 \times 10^{6} \mathrm{CFU} / \mathrm{mL}, 2 \times 10^{6}\right.$ $\mathrm{CFU} / \mathrm{mL}$, and $4 \times 10^{6} \mathrm{CFU} / \mathrm{mL}$ ) and placed in a nebulizer within an aerosol exposure chamber that was custom-made by the University of Wisconsin. In preliminary studies, groups of 3 mice were exposed to the aerosol at each concentration for 15 minutes. After 24 hours, the mice were euthanized, and homogenized lungs were plated on 7H10 agar plates supplemented with OADC. CFUs were counted after 14-22 days of incubation at $37^{\circ} \mathrm{C}$. The concentration that deposited approximately $75-100$ bacteria in the lung during aerosol infection was used for further studies (Supplemental Table 5).

Lung cell preparation. Lungs were harvested from the control and Mtb-infected WT C57BL/6 and Thra $1^{\mathrm{PV} /+}$ mice 30 days after Mtb challenge and were placed into $60 \mathrm{~mm}$ dishes containing $2 \mathrm{~mL}$ of HBSS. The tissues were minced with scissors into pieces no larger than $2-3 \mathrm{~mm}$, and the fluid was discharged onto a $70 \mu \mathrm{m}$ filter (BD Biosciences) that had been prewetted with $1 \mathrm{~mL}$ of PBS containing $0.5 \%$ BSA (Sigma-Aldrich) suspended over a 50-mL conical tube. The syringe plunger was then used to gently disrupt the lung tissue before washing the filter with $2 \mathrm{~mL}$ of cold PBS $/ 0.5 \%$ BSA. The total number of viable cells in the lungs was determined with the trypan blue exclusion method. For flow cytometry experiments, we gated the total lung CD $45^{+}$cells (leukocytes) and measured various cell populations.

Flow cytometry and intracellular staining. After the mice were euthanized, lungs were perfused with $5 \mathrm{~mL}$ of PBS via the left ventricle. Lungs were mechanically homogenized and passed through a $70 \mu \mathrm{m}$ cell strainer. Remaining RBCs were lysed using BD Pharm Lyse (BD Biosciences). Cells were treated with an Fc blocker (TruStain, BioLegend) prior to staining. Surface staining to identify leukocyte populations was then performed. For FoxP3 intracellular staining, cells were permeabilized with $0.1 \%$ saponin and stained for intracellular FoxP3. The cells were washed, resuspended in FACS buffer, and analyzed by using an Attune flow cytometer.

Multiplex ELISA. Mouse multiplex ELISA kits (36-Plex kits, Thermo Fisher Scientific) were used to measure chemokine and cytokine levels according to the manufacturer's instructions.

Histopathology of necrotic lesions in the lung. At the specified time points, mice were euthanized, and the harvested lungs were placed in 10\% neutral buffered formalin (StatLab) for 48 hours to inactivate the infectious agent. Paraffin-embedded blocks were cut into $5 \mu \mathrm{m}$ thick sections. For morphometric lesion analyses, the lung sections were stained with $\mathrm{H} \& \mathrm{E}$ and examined in a blinded manner to assess the necrotic lesions as previously described (72). Briefly, each lung lobe was quantified for the lesion area and percentage of lung lesions by using ImageJ (NIH). Two investigators, DT and RR, independently assessed the immunohistochemical readouts using morphometric analyses.

Statistics used for mouse studies. Prism 7 (GraphPad) was used for statistical analyses. The results are shown as the mean \pm SEM. Comparisons between groups were performed by a paired or unpaired $t$ test, as appropriate. Mouse survival was compared using the Kaplan-Meier log-rank test.

Mouse study approval. The IACUC of the University of Texas Health Science Center approved all the animal studies. Animal procedures involving the care and use of mice were performed in accordance with the guidelines of the NIH/OLAW (Office of Laboratory Animal Welfare).

\section{Author contributions}

RV and VLV devised the project and the main conceptual ideas, proofed the outline, and conceived and planned the experiments. KPD, DT, and VSKN planned and carried out the experiments, performed the analysis, and designed the figures. PP and RKR carried out the experiments. KPS, DT, and KPD performed data analysis. SYC and SP provided Thra $1 \mathrm{PV}^{/+}$mice. MSA screened the patients and was involved in participant counseling, recruitment, and follow-up. MJ, RTNM, MGN, and SYC reviewed and edited the manuscript. DT, RV, and KPD drafted the manuscript. The order of co-senior authorship was based on the contribution of main conceptual idea, experimental designing, and drafting of manuscript.

\section{Acknowledgments}

We thank the NIH, CRDF Global, the Cain Foundation for Infectious Disease Research, and the Department 
of Pulmonary Immunology and the Department of Biotechnology, New Delhi, India, for providing financial support. This work was supported by grants from the NIH (AI123310 to RV), CRDF Global, the Cain Foundation for Infectious Disease Research, the Department of Pulmonary Immunology, and the Department of Biotechnology (BT/PR9622/MED/15/109/2013 to VV), New Delhi, India.

Address correspondence to: Ramakrishna Vankayalapati, Department of Pulmonary Immunology, University of Texas Health Science Center at Tyler, 11937 US Highway 271, Tyler, Texas 75708, USA. Phone: 903.877.5190; Email: krishna.vankayalapati@uthct.edu. Or to: Vijaya Lakshmi Valluri, Immunology and Molecular Biology Department, Bhagwan Mahavir Medical Research Centre, Hyderabad 500004, India. Phone: 9140.2349.7306 ext. 306; Email: vijayavalluri@gmail.com.

1. WHO. Global Tuberculosis Report 2020. https://www.who.int/publications/i/item/9789240013131. Accessed May $28,2021$.

2. WHO. Global Tuberculosis Report 2018. https://apps.who.int/iris/bitstream/handle/10665/274453/9789241565646-eng.pdf. Accessed May 28, 2021.

3. Dye C. Global epidemiology of tuberculosis. Lancet. 2006;367(9514):938-940.

4. Menzies D, et al. Four months of rifampin or nine months of isoniazid for latent tuberculosis in adults. $N$ Engl J Med. 2018;379(5):440-453.

5. Arguello Perez E, et al. Management of latent tuberculosis infection among healthcare workers: 10-year experience at a single center. Clin Infect Dis. 2017;65(12):2105-2111.

6. Sester M, et al. Challenges and perspectives for improved management of HIV/Mycobacterium tuberculosis co-infection. Eur Respir J. 2010;36(6):1242-1247.

7. Zumla A, et al. Impact of HIV infection on tuberculosis. Postgrad Med J. 2000;76(895):259-268.

8. Wallis RS. Tumour necrosis factor antagonists: structure, function, and tuberculosis risks. Lancet Infect Dis. 2008;8(10):601-611.

9. Brassard P, et al. Antirheumatic drugs and the risk of tuberculosis. Clin Infect Dis. 2006;43(6):717-722.

10. Rangaka MX, et al. Predictive value of interferon- $\gamma$ release assays for incident active tuberculosis: a systematic review and meta-analysis. Lancet Infect Dis. 2012;12(1):45-55.

11. Walzl G, et al. Immunological biomarkers of tuberculosis. Nat Rev Immunol. 2011;11(5):343-354.

12. Lawn SD, et al. Advances in tuberculosis diagnostics: the Xpert MTB/RIF assay and future prospects for a point-of-care test. Lancet Infect Dis. 2013;13(4):349-361.

13. Luo Y, et al. Early progression to active tuberculosis is a highly heritable trait driven by $3 \mathrm{q} 23$ in Peruvians. Nat Commun. 2019;10(1):3765.

14. Zak DE, et al. A blood RNA signature for tuberculosis disease risk: a prospective cohort study. Lancet. 2016;387(10035):2312-2322

15. Suliman S, et al. Four-gene pan-African blood signature predicts progression to tuberculosis. Am J Respir Crit Care Med. 2018;197(9):1198-1208.

16. Warsinske $\mathrm{H}$, et al. Host-response-based gene signatures for tuberculosis diagnosis: a systematic comparison of 16 signatures. PLoS Med. 2019;16(4):e1002786.

17. Roe J, et al. Blood transcriptomic stratification of short-term risk in contacts of tuberculosis. Clin Infect Dis. 2020;70(5):731-737.

18. Leong S, et al. Cross-validation of existing signatures and derivation of a novel 29-gene transcriptomic signature predictive of progression to TB in a Brazilian cohort of household contacts of pulmonary TB. Tuberculosis (Edinb). 2020;120:101898.

19. Penn-Nicholson A, et al. RISK6, a 6-gene transcriptomic signature of TB disease risk, diagnosis and treatment response. Sci Rep. 2020;10(1):8629.

20. Weiner J, et al. Metabolite changes in blood predict the onset of tuberculosis. Nat Commun. 2018;9(1):5208.

21. Petruccioli E, et al. Correlates of tuberculosis risk: predictive biomarkers for progression to active tuberculosis. Eur Respir J. 2016;48(6):1751-1763.

22. Stein CM, et al. Resistance and susceptibility to Mycobacterium tuberculosis infection and disease in tuberculosis households in Kampala, Uganda. Am J Epidemiol. 2018;187(7):1477-1489.

23. Fox GJ, et al. Household-contact investigation for detection of tuberculosis in Vietnam. NEngl J Med. 2018;378(22):2141.

24. Laghari M, et al. Contact screening and risk factors for TB among the household contact of children with active TB: a way to find source case and new TB cases. BMC Public Health. 2019;19(1):1274.

25. Fox GJ, et al. Contact investigation for tuberculosis: a systematic review and meta-analysis. Eur Respir J. 2013;41(1):140-156.

26. Saunders MJ, et al. A household-level score to predict the risk of tuberculosis among contacts of patients with tuberculosis: a derivation and external validation prospective cohort study. Lancet Infect Dis. 2020;20(1):110-122.

27. Cooper AM. Cell-mediated immune responses in tuberculosis. Annu Rev Immunol. 2009;27:393-422.

28. Cooper AM, Khader SA. The role of cytokines in the initiation, expansion, and control of cellular immunity to tuberculosis. Immunol Rev. 2008;226:191-204.

29. Flynn JL, et al. Tumor necrosis factor-alpha is required in the protective immune response against Mycobacterium tuberculosis in mice. Immunity. 1995;2(6):561-572.

30. Ardain A, et al. Group 3 innate lymphoid cells mediate early protective immunity against tuberculosis. Nature. 2019;570(7762):528-532.

31. Shafiani S, et al. Pathogen-specific regulatory T cells delay the arrival of effector T cells in the lung during early tuberculosis. J Exp Med. 2010;207(7):1409-1420.

32. Cohen SB, et al. Alveolar macrophages provide an early Mycobacterium tuberculosis niche and initiate dissemination. Cell Host Microbe. 2018;24(3):439-446.

33. Venkatasubramanian S, et al. IL-21-dependent expansion of memory-like NK cells enhances protective immune responses 
against Mycobacterium tuberculosis. Mucosal Immunol. 2017;10(4):1031-1042.

34. Aibana O, et al. Vitamin D status and risk of incident tuberculosis disease: a nested case-control study, systematic review, and individual-participant data meta-analysis. PLoS Med. 2019;16(9):e1002907.

35. Gou X, et al. The association between vitamin D status and tuberculosis in children: a meta-analysis. Medicine (Baltimore). 2018;97(35):e12179

36. Pan S-C, et al. Effect of diabetes on tuberculosis control in 13 countries with high tuberculosis: a modelling study. Lancet Diabetes Endocrinol. 2015;3(5):323-330.

37. Lurie MB, et al. On the role of the thyroid in native resistance to tuberculosis. I. The effect of hyperthyroidism. Am Rev Tuberc 1959;79(2):152-179.

38. Bartlett JA, et al. Immune function in healthy adolescents. Clin Diagn Lab Immunol. 1998;5(1):105-113.

39. van der Spek AH, et al. Regulation of intracellular triiodothyronine is essential for optimal macrophage function. Endocrinology. 2018;159(5):2241-2252.

40. Kwakkel J, et al. A novel role for the thyroid hormone-activating enzyme type 2 deiodinase in the inflammatory response of macrophages. Endocrinology. 2014;155(7):2725-2734.

41. Kaneshige $M$, et al. A targeted dominant negative mutation of the thyroid hormone $\alpha 1$ receptor causes increased mortality, infertility, and dwarfism in mice. Proc Natl Acad Sci U S A. 2001;98(26):15095.

42. Bloom BR, et al. Tuberculosis. In: Holmes KK, et al, eds. Major Infectious Diseases 3rd Edition. World Bank Publications; 2017: Chapter 11.

43. Imtiaz S, et al. Alcohol consumption as a risk factor for tuberculosis: meta-analyses and burden of disease. Eur Respir J. 2017;50(1):1700216.

44. Padrão E, et al. Tuberculosis and tobacco: is there any epidemiological association? Eur Respir J. 2018;51(1):1702121.

45. Lee PH, et al. Glycemic control and the risk of tuberculosis: a cohort study. PLoS Med. 2016;13(8):e1002072.

46. Donald PR, et al. Age and the epidemiology and pathogenesis of tuberculosis. Lancet. 2010;375(9729):1852-1854

47. Martinez L, et al. The risk of tuberculosis in children after close exposure: a systematic review and individual-participant meta-analysis. Lancet. 2020;395(10228):973-984.

48. Vanden Driessche K, et al. Immune vulnerability of infants to tuberculosis. Clin Dev Immunol. 2013;2013:781320.

49. Guerra-Laso JM, et al. Macrophages from elders are more permissive to intracellular multiplication of Mycobacterium tuberculosis. Age (Dordr). 2013;35(4):1235-1250.

50. Zelner JL, et al. Age-specific risks of tuberculosis infection from household and community exposures and opportunities for interventions in a high-burden setting. Am J Epidemiol. 2014;180(8):853-861.

51. Wira CR, et al. The role of sex hormones in immune protection of the female reproductive tract. Nat Rev Immunol. 2015;15(4):217-230

52. Chavarria-Smith J, et al. Humoral immunity goes hormonal. Nat Immunol. 2018;19(10):1044-1046.

53. Webster JI, et al. Neuroendocrine regulation of immunity. Annu Rev Immunol. 2002;20(1):125-163.

54. Cain DW, Cidlowski JA. Immune regulation by glucocorticoids. Nat Rev Immunol. 2017;17(4):233-247.

55. Kleynhans L, et al. Changes in host immune-endocrine relationships during tuberculosis treatment in patients with cured and failed treatment outcomes. Front Immunol. 2017;8:690.

56. Lurie $\mathrm{MB}$, et al. On the role of the thyroid in native resistance to tuberculosis. II. The effect of hypothyroidism; the mode of action of thyroid hormones. Am Rev Tuberc. 1959;79(2):180-203.

57. Hoermann R, Midgley JE, Larisch R, Dietrich JW. Homeostatic Control of the Thyroid-Pituitary Axis: Perspectives for Diagnosis and Treatment. Front Endocrinol (Lausanne). 2015;6:177.

58. Mayer-Barber KD, et al. Innate and adaptive interferons suppress IL- $1 \alpha$ and IL- $1 \beta$ production by distinct pulmonary myeloid subsets during Mycobacterium tuberculosis infection. Immunity. 2011;35(6):1023-1034.

59. Di Paolo NC, et al. Interdependence between interleukin-1 and tumor necrosis factor regulates TNF-dependent control of Mycobacterium tuberculosis infection. Immunity. 2015;43(6):1125-1136.

60. Perrotta C, et al. The thyroid hormone triiodothyronine controls macrophage maturation and functions: protective role during inflammation. Am J Pathol. 2014;184(1):230-247.

61. Garand M, et al. Functional and phenotypic changes of natural killer cells in whole blood during Mycobacterium tuberculosis infection and disease. Front Immunol. 2018;9:257.

62. Vankayalapati R, et al. Role of NK cell-activating receptors and their ligands in the lysis of mononuclear phagocytes infected with an intracellular bacterium. J Immunol. 2005;175(7):4611-4617.

63. Lanier LL. NK cell receptors. Annu Rev Immunol. 1998;16(1):359-393.

64. Esaulova E, et al. The immune landscape in tuberculosis reveals populations linked to disease and latency. Cell Host Microbe. 2021;29(2):165-178.

65. Sakaguchi S, et al. FOXP3+ regulatory T cells in the human immune system. Nat Rev Immunol. 2010;10(7):490-500

66. O'Garra A, et al. IL-10-producing and naturally occurring CD4+ Tregs: limiting collateral damage. J Clin Invest. 2004;114(10):1372-1378.

67. Boer MC, et al. Regulatory T-cells at the interface between human host and pathogens in infectious diseases and vaccination. Front Immunol. 2015;6:217.

68. Lin PL, et al. CD4 T cell depletion exacerbates acute Mycobacterium tuberculosis while reactivation of latent infection is dependent on severity of tissue depletion in cynomolgus macaques. AIDS Res Hum Retroviruses. 2012;28(12):1693-1702.

69. Cooper AM, et al. Role of innate cytokines in mycobacterial infection. Mucosal Immunol. 2011;4(3):252-260.

70. Jara EL, et al. Modulating the function of the immune system by thyroid hormones and thyrotropin. Immunol Lett. $2017 ; 184: 76-83$.

71. Devalraju KP, et al. IL-17 and IL-22 production in HIV+ individuals with latent and active tuberculosis. BMC Infect Dis. 2018;18(1):321

72. Tripathi D, et al. IL-22 produced by type 3 innate lymphoid cells (ILC3s) reduces the mortality of type 2 diabetes mellitus (T2DM) mice infected with Mycobacterium tuberculosis. PLoS Pathog. 2019;15(12):e1008140. 